\title{
Harmonic forms and near-minimal singular foliations
}

\author{
Gabriel Katz
}

\begin{abstract}
For a closed 1-form $\omega$ with Morse singularities, Calabi discovered a simple global criterion for the existence of a Riemannian metric in which $\omega$ is harmonic. For a codimension 1 foliation $\mathcal{F}$, Sullivan gave a condition for the existence of a Riemannian metric in which all the leaves of $\mathcal{F}$ are minimal hypersurfaces. The conditions of Calabi and Sullivan are strikingly similar. If a closed form $\omega$ has no singularities, then both criteria are satisfied and, for an appropriate choice of metric, $\omega$ is harmonic and the associated foliation $\mathcal{F}_{\omega}$ is comprised of minimal leaves. However, when $\omega$ has singularities, the foliation $\mathcal{F}_{\omega}$ is not necessarily minimal.

We show that the Calabi condition enables one to find a metric in which $\omega$ is harmonic and the leaves of the foliation are minimal outside a neighborhood $U$ of the $\omega$-singular set. In fact, we prove the best possible result of this type: we construct families of metrics in which, as $U$ shrinks to the singular set, the taut geometry of the foliation $\mathcal{F}_{\omega}$ outside $U$ remains stable. Furthermore, all compact leaves missing $U$ are volume minimizing cycles in their homology classes. Their volumes are controlled explicitly.
\end{abstract}

Mathematics Subject Classification (2000). 51 (Geometry), 58 (Global Analysis, Analysis on Manifolds).

Keywords. Closed 1-forms, intrinsic harmonicity, minimal foliations, volume-minimizing cycles, Morse-type singularities.

\section{Introduction}

Probably, it has been observed for a long time that a single simple geometric property implies two quite different phenomena: the first is the intrinsic harmonicity of a given closed 1-form $\omega$ and the second - the intrinsic minimality of the foliation $\mathcal{F}_{\omega}$ determined by this form. The first implication of this global geometric property (we call it the Calabi Property) was discovered by Calabi ([Ca]) and the second, for non-singular foliations, - by Sullivan ([S2]). It is easy to see that, if $\omega$ has no singularities, then the Calabi property is satisfied and, as a result, $\omega$ is intrinsically harmonic and $\mathcal{F}_{\omega}$ is intrinsically minimal. However, 1-forms typically do have singularities and so do the corresponding foliations. In general, the $\omega$-singularities obstruct both the harmonicity of $\omega$ and the minimality of $\mathcal{F}_{\omega}$.

The research has been supported by US-Israel Binational Science Foundation Grant 9400073. 
Therefore, it is natural to ask whether, in the presence of the Calabi property, there exists a Riemannian metric with respect to which $\omega$ is harmonic and the leaves of $\mathcal{F}_{\omega}$ are volume-minimizing hypersurfaces. In this paper we show that one can find such a metric which harmonizes $\omega$ and "almost" minimizes the leaves of $\mathcal{F}_{\omega}$, thus bringing Calabi's and Sullivan's theories under a single roof.

Let us clarify and expand upon this claim. Let $M$ be a smooth compact $n$ manifold and $\omega-$ a closed 1 -form on $M$. We say that $\omega$ is of Morse type if, locally, it is the differential of a Morse function. We assume that $\omega$ has no singularities on the boundary $\partial M$ and that its restriction on $\partial M$ also is a form of the Morse type.

Let $S_{\omega}$ denote the singular set of $\omega$-it is a finite collection of points. Similarly, let $S_{\omega}^{\partial}$ denote the singular set of $\omega$, restricted to $\partial M$. Put

$$
S_{\omega}^{\star}=S_{\omega} \sqcup S_{\omega}^{\partial}
$$

and

$$
M_{\omega}^{\circ}=M \backslash S_{\omega}, \quad \partial M_{\omega}^{\circ}=\partial M \backslash S_{\omega}^{\partial}, \quad M_{\omega}^{\star}=M \backslash S_{\omega}^{\star} .
$$

Definition 0.1. We say that a smooth path $\gamma:[0,1] \rightarrow M$ is $\omega$-positive if, for any $t \in[0,1], \omega(\dot{\gamma}(t))>0$. Here $\dot{\gamma}(t)$ denotes the velocity vector tangent to $\gamma$ at $\gamma(t)$

The following is a modification of an important global property of $\omega$, studied in $[\mathrm{Ca}]$.

Definition 0.2. We say that $\omega$ satisfies the relative Calabi property, if

(i) for each point $x \in M_{\omega}^{\circ}$, there exists through $x$ a closed $\omega$-positive path;

(ii) for each point $x \in \partial M_{\omega}^{\circ}$, there exists in $\partial M$ through $x$ a closed $\omega$-positive path.

Calabi was investigating the following problem: Given a closed 1-form $\omega$ on a closed smooth $n$-manifold $M$, when does there exist a Riemannian metric $g$, such that $\omega$ is harmonic with respect to the $g$ ?

If such a metric exists, we will say that $\omega$ is intrinsically harmonic.

In this paper the harmonicity of a closed differential form $\omega$ for manifolds with boundary is interpreted as the property $d(* \omega)=0$, where " $*$ " stands for the Hodge star-operator (and not as a general solution of the Laplace's equation).

Calabi proved that, for a closed $M$, condition (i) is equivalent to the intrinsic harmonicity of $\omega([\mathrm{Ca}])$.

Remark. Locally, $\omega=d f$, $f$ being a Morse function. Property (i) prevents $f$ from having local maxima and minima in the interior of $M$ and (ii) - on its boundary.

A closed Morse-type 1-form which possesses properties (i) and (ii) will be called a Calabi form. 
Since $\omega$ is closed, it defines a $(n-1)$-dimensional foliation $\mathcal{F}_{\omega}$ on $M$. This foliation may have singular leaves with the Morse-type singularities: locally, in the leaf topology, the $\mathcal{F}_{\omega}$-leaves are the hypersurfaces of constant level for a function $f$ whose differential is $\omega$. Hence, in a neighborhood of a singularity, the singular leaves are homeomorphic to cones over products of two spheres.

It is possible to verify that Calabi's properties (i), (ii) have the following nice interpretation in terms of the foliation $\mathcal{F}_{\omega}$ :

Each compact leaf component is pierced by a loop transversal to $\mathcal{F}_{\omega}$ and a similar property holds for $\left.\mathcal{F}_{\omega}\right|_{\partial M}$.

The verification depends on two observations:

1 ) if there exists an $\omega$-positive loop through a nonsingular point $x$ of a leaf component $\mathcal{L}$, then such a loop exits through any other nonsingular point $y \in \mathcal{L}$;

2 ) any non-compact leaf component $\mathcal{L}$ is pierced by an $\omega$-positive loop (cf. [FKL]).

For foliations without singularities on closed manifolds, Sullivan showed ([S2]), that property $(0.2)$ is equivalent to the existence of a metric in which all the leaves are minimal hypersurfaces. In other words, (0.2), equivalent to the Calabi property of Definition 0.2, implies the intrinsic minimality of the leaves. Such intrinsically minimal foliations are also called taut. They have been extensively studied by a number of authors: [S1], [S2], [KT] [HL], [HT].

In general, the foliation $\mathcal{F}_{\omega}$ fails to be intrinsically minimal in the vicinity of its singularity. For example, consider the 1-dimensional foliation on the plane in a neighborhood of a hyperbolic singularity; clearly, there is no metric in which all the hyperbolas are geodesic curves. Therefore, in general, even for Calabi forms, one can not expect $\mathcal{F}_{\omega}$ to be minimal. However, the next best to the minimality property can be achieved: the deviation of the leaves from minimality numerically can be made arbitrary small and arbitrary localized in the vicinity of the singular set $S_{\omega}^{\star}$. Such foliations will be called near-minimal.

Our goal is to merge the Sullivan's and Calabi's results within a more general context of singular foliations. In particular, we going to prove the following fact:

Given a Calabi form $\omega$, there exists a metric, with respect to which the form is harmonic and all the leaves of $\mathcal{F}_{\omega}$ are near-minimal (cf. Theorem A).

Furthermore, we show that the orthogonal to $\mathcal{F}_{\omega}$ 1-foliation $\mathcal{F}_{* \omega}$ (tangent to the kernels of the form $* \omega$ ) also is near-minimal: it consists of leaves that deviate from geodesic lines only in the vicinity of the $\omega$-singular points. Again, by the choice of metric, the length deviation can be made arbitrary small.

This gives a beautiful geometric structure: an intrinsically harmonic 1-form produces a pair of mutually orthogonal singular foliations, both of which are nearminimal and, actually, minimal away of the singularities. 
In small dimensions, these foliations definitely fail to be minimal with respect to any metric. Nevertheless, we suspect that, if the dimension of $M$ exceeds 7 , sometimes the word "near" can be be dropped from the statement about the leaves of $\mathcal{F}_{\omega}$ (cf. Conjecture 4.1).

Many results of this paper on the volume-minimizing cycles (built of compact $\mathcal{F}_{\omega}$-leaves) have somewhat more general classical analogs, formulated in terms the mass-minimizing foliation cycles and currents. These classical results were established for generic non-singular foliations on compact closed manifolds (cf. [HL], [S], [S1]) and stated in terms of the Geometric Measure Theory. In this context, our contribution can be described as reproducing these results for the non-compact manifolds $\left\{M_{\omega}^{\star}\right\}$ in a fashion that permits an extension of the metric in question across the singularities. In particular, we prove (cf. Proposition 2.5) a "taut" version of the rational Poincaré duality (in dimensions 1 and $(n-1)$ ).

We also extend the setting for manifolds with boundary and investigate the impact of boundary effects on the intrinsic harmonicity and minimality of the leaves. When the form $\left.\omega\right|_{\partial M}$ is non-singular, and $\omega$ satisfies $(0.2)$, then it is possible to "synchronize" the harmonicity and minimality on $M$ and $\partial M$; for $\left.\omega\right|_{\partial M}$ with singularities, it is very much an open problem.

The introduction of foliations with singularities, induced by closed 1-forms, drastically changes the landscape of the classical foliation theory. On the one hand, one avoids some of the pathologies (think about the Reeb foliation), characteristic for the most general (non-singular) foliations, on the other hand, the presence of singularities generates new diverse possibilities and complications. For example, Novikov's Theorem $([\mathrm{N} 2])$ states that, if a foliation $\mathcal{F}_{\omega}$ is produced by a closed non-singular form $\omega$, then all the leaves are either compact, or non-compact. This is not the case for foliations generated by closed forms with singularities - such foliations often are mixed bags.

The objects and constructions that facilitate our proofs are, so to speak, handmade. As a result, we are able to avoid a great deal of Functional Analysis and Geometric Measure Theory. Our only generic tool is Stokes' Theorem.

\section{Statements of the main results}

Most of our results are organized in eight big blocks: Theorems $\mathrm{A}, \mathrm{B}, \mathrm{A}^{\perp}, \mathrm{B}^{\perp}$ from Section 1, and Theorems C, D, $\mathrm{C}^{\perp}, \mathrm{D}^{\perp}$ from Section 3. Theorems A, B, $\mathrm{A}^{\perp}, \mathrm{B}^{\perp}$ deal with more restrictive boundary conditions imposed on the 1 -form $\omega$ (cf. Definition 0.2), while Theorems C, D, $\mathrm{C}^{\perp}, \mathrm{D}^{\perp}$ - with more relaxed ones (cf. Definition 3.1). Theorems A, B, C, D are concerned mostly with the $(n-1)$ dimensional foliation $\mathcal{F}_{\omega}$, while Theorems $\mathrm{A}^{\perp}, \mathrm{B}^{\perp}, \mathrm{C}^{\perp}, \mathrm{D}^{\perp}-$ with an auxiliary 1-dimensional foliation $\mathcal{F}_{\Omega}$, transversal to $\mathcal{F}_{\omega}$ and generated by the gradient flow 
of $\omega$. Otherwise, the theorems share many similar (and dual) claims that we have chosen to repeat in order to avoid confusing multiple cross-references.

Prior to formulating a number of propositions, we shall introduce a few notations and make a few comments.

For each $\omega$-singular point $A_{j} \in S_{\omega}$, there exists a system of Morse coordinates $\left\{x_{i}=x_{i}(j)\right\}_{1 \leq i \leq n}$, centered on $A_{j}$, so that, locally,

$$
\omega=\sum_{i=1}^{n} a_{i} x_{i} d x_{i} \quad a_{i} \in \mathbf{R} .
$$

Since Calabi's property prevents the $a_{i}$ 's from all being of the same sign, it is possible to stretch the coordinates $\left\{x_{i}\right\}$ in such a way that

$$
\sum_{i=1}^{n} a_{i}=0
$$

This calibration of $\left\{x_{i}\right\}$ insures that $\omega$ is harmonic with respect to the euclidean metric $\left(d g_{E}\right)^{2}=\sum_{i=1}^{n} d x_{i}^{2}$.

A similar choice of Morse coordinates is available at the singularities of $\left.\omega\right|_{\partial M}$. In a neighborhood of a singularity $A_{k}^{\partial} \in S_{\omega}^{\partial}$,

$$
\omega=a_{1} d x_{1}+\sum_{i=2}^{n} a_{i} x_{i} d x_{i}
$$

where $x_{1}$ is a coordinate in a transversal to the boundary direction, so that $x_{1}$ is positive in $M$ and $\left.\omega\right|_{\partial M}=\sum_{i=2}^{n} a_{i} x_{i} d x_{i}$. Due to property (ii) in Definition 0.2 , we may assume that $\sum_{i=2}^{n} a_{i}=0$ to insure the harmonicity of $\left.\omega\right|_{\partial M}$ in the euclidean metric $\left(\left.d g_{E}\right|_{\partial M}\right)^{2}=\sum_{i=2}^{n} d x_{i}^{2}$.

In fact, the singularities of $\left.\omega\right|_{\partial M}$ come in two flavors: positive with a positive $a_{1}$ 's and negative with a negative $a_{1}$ 's. Thus, $S_{\omega}^{\partial}=S_{\omega,+}^{\partial} \sqcup S_{\omega,-}^{\partial}$, where $S_{\omega,+}^{\partial}$ stands for the set of the positive singularities.

Notice that, for odd $n$ 's, the condition $\sum_{i=1}^{n} a_{i}=0$ prevents us from calibrating Morse coordinates, so that $\left|a_{i}\right|=1$ for all $i$ 's. Nevertheless, without loss of generality, one can assume that the modules of all $a_{i}$ 's are equal 1 or 2 . The same applies to the singularities from $S_{\omega}^{\partial}$. For those we can assume that $\left|a_{1}\right|=1$ and the rest of the $\left|a_{i}\right|$ 's are equal 1 or 2 . Unless, stated differently, these assumptions will hold in what follows.

Of course, the topology of $M$ imposes restrictions on the list of singularities from $S_{\omega}$ and $S_{\omega}^{\partial}$ (for example, see [N], [N1]). On the other hand, the Calabi property itself does not restrict the types of $\omega$-singularities in $M$, besides the obvious exclusion of the singular points of indices 0 and $n$. In fact, given a closed 1 -form $\omega$ on a closed manifold with no singularities of indices 0 and $n$, it is possible 
to deform it to a new closed form $\omega^{\prime}$ with the same list of singular points and satisfying Calabi's condition $([\mathrm{FKL}]),([\mathrm{Ho}])$.

For any $\epsilon>0$, consider the $\epsilon$-ellipsoids

$$
B_{\epsilon, j}=\left\{\left\{x_{i}\right\}: \quad \sum_{i=1}^{n} a_{i}^{2} x_{i}^{2}<\epsilon^{2}\right\}
$$

in the calibrated Morse coordinates $\left\{x_{i}\right\}$ around each of the singularities $A_{j} \in S_{\omega}$. Similarly, for any $\epsilon>0$ and each singularity $A_{k}^{\partial} \in S_{\omega}^{\partial}$, consider the half-ellipsoid

$$
B_{\epsilon, k}^{+}=\left\{\left\{x_{i}\right\}: \quad a_{1}^{2} x_{1}^{2}+\sum_{i=2}^{n} a_{i}^{2} x_{i}^{2}<\epsilon^{2}, \quad x_{1} \geq 0\right\}
$$

(The ellipsoids $\left\{B_{\epsilon, j}\right\}_{j}$ and $\left\{B_{\epsilon, k}^{+}\right\}_{k}$ will serve as elements of a special $\omega$-subordinate cover of $M$.)

In what follows we always assume that $\epsilon$ is sufficiently small, so that all the $2 \epsilon$-ellipsoids $\left\{B_{2 \epsilon, j}\right\}_{j}$ and $\left\{B_{2 \epsilon, k}^{+}\right\}_{k}$ are disjoint.

We denote by $M_{\omega, \epsilon}^{\circ}$ the complement in $M$ to the $\epsilon$-ellipsoids, centered on the points from $S_{\omega}$. Similarly, let $M_{\omega, \epsilon}^{\star}$ denote the complement to the $\epsilon$-ellipsoids centered on the points from $S_{\omega}^{\star}$.

We say that a differential $i$-form $\Omega$ vanishes on the boundary $\partial M$, if it gets zero values on any $i$-tuple of vectors, tangent to $\partial M$.

Consider the natural pairing

$$
\star: \quad H^{i}(M, \partial M ; \mathbf{R}) \otimes H_{i}(M, \partial M ; \mathbf{R}) \longrightarrow \mathbf{R} .
$$

For a closed $i$-form $\Omega$ which vanishes on $\partial M$ and a relative $i$-cycle $\Sigma$, realized by an embedding $(\Sigma, \partial \Sigma) \hookrightarrow(M, \partial M)$ of a pseudo-manifold ${ }^{1} \Sigma$, the pairing $\star$ is given by integration:

$$
[\Omega] \star[\Sigma]=\int_{\Sigma} \Omega .
$$

Furthermore, if the restriction of $\Omega$ on $\partial M$ is exact, i.e. if $\left.\Omega\right|_{\partial M}=\mathrm{d} \Psi$ for an $(i-1)$-form $\Psi$, and $\Psi$ is supported on a set $A \subset \partial M$ which retracts on a $(n-i-1)$-dimensional CW-complex, then the integration of $\Omega$ produces a welldefined functional on $H_{i}(M, \partial M ; \mathbf{Z})$ and thus, on $H_{i}(M, \partial M ; \mathbf{R})$.

Let $*_{g}$ denote the Hodge star-operator in a Riemannian metric $g$.

\footnotetext{
1 a CW-complex with a singular set of codimension 2 .
} 
Finally, we are in position to formulate

Theorem A. Let $M$ be an oriented smooth and compact $n$-manifold equipped with a Calabi 1-form $\omega$ [cf. Def. 0.2 or (0.2)]. Given positive numbers $\lambda, \mu, \lambda \geq 1,{ }^{2}$ there exists a smooth $\lambda \mu$-family of Riemannian metrics $g_{\lambda, \mu}$ on $M$, so that the following holds:

(1) All the metrics $g_{\lambda, \mu}$ are conformally equivalent. For any $\mu^{\prime} \leq \mu$, the metrics $g_{\lambda, \mu}$ and $g_{\lambda, \mu^{\prime}}$ coincide on the complement to the $\mu$-ellipsoids centered on the singularities of $\omega$.

(2) The form $\omega$ is harmonic with respect to $g_{\lambda, \mu}$.

(3) The closed $(n-1)$-form $\Omega=*_{g_{\lambda, \mu}}(\omega)$ is $\lambda, \mu$-independent. It gives rise to a non-trivial class $[\Omega] \in H^{n-1}(M, \partial M ; \mathbf{R})$.

(4) All the leaves of the foliation $\mathcal{F}_{\omega}$, outside of the $\mu$-ellipsoids, are minimal hypersurfaces ${ }^{3}$ in the metric $g_{\lambda, \mu}$.

(5) If the restriction $\left.\omega\right|_{\partial M}$ has no singularities in $\partial M$, then $\left.\omega\right|_{\partial M}$ has analogous properties (1)-(4) with respect to the $g_{\lambda, \mu}$-induced metric on the boundary.

Statement (2) above is a slight reenforcement of the main result in [Ca]; (4) should be compared with [S2], Corollary 3, which deals with non-singular foliations. The fact that there exists a metric, for which both statements (2) and (4) are valid, is a new observation even for a non-singular $\omega$.

Theorem B. Let $M, \omega, \Omega$ and $g_{\lambda, \mu}$ be as in Theorem $A$.

(1) Let $F$ be any finite union of compact leaves of the foliation $\mathcal{F}_{\omega}$, which does not intersect the $\mu$-ellipsoids around the singularities of $\omega$. Then $F$ minimizes the $g_{\lambda, \mu}$-induced $(n-1)$-volume $V(F)$ among all relative cycles (pseudo-manifolds) $(\Sigma, \partial \Sigma) \hookrightarrow(M, \partial M)$, subject to the homological constraint $[\Omega] \star[\Sigma]=[\Omega] \star[F]$. In particular, $F$ minimizes the volume in its relative homology class. This minimal volume $V(F)=\lambda^{-1}[\Omega] \star[F]$.

(2) Any relative cycle $(\Sigma, \partial \Sigma) \hookrightarrow(M, \partial M)$ of the volume $V(F)$ and such that $[\Omega] \star[\Sigma]=[\Omega] \star[F]$, outside of the $\mu$-ellipsoids, is comprised of compact leaves of the foliation $\mathcal{F}_{\omega}$ (being restricted to the exterior of the ellipsoids).

The volume of the portion $\Sigma_{\mu}^{S}$ of $\Sigma$, lying inside of the $\mu$-ellipsoids, is given by the integral $\lambda^{-1} \int_{\Sigma_{\mu}^{S}} \Omega$. When $\lambda \rightarrow+\infty$, it declines as $\sim \lambda^{-1}$; when $\mu \rightarrow 0$, it declines as $\sim \mu^{n-1}$.

(3) For any union $\tilde{F}$ of compact leaf components homologous to $F$, the volume variation $|V(\tilde{F})-V(F)| \leq K \cdot \mu^{n-1}$, where $K$ is a positive, $\lambda \mu$-independent

\footnotetext{
2 The role of parameter $\lambda$ will be reveled in Theorem B. In fact, all the claims are valid for any $\lambda>\max _{k}\left|a_{1}(k)\right|$.

3 i.e. any compactly supported leaf perturbation in $M_{\omega, \mu}^{\star}$, fixed on the boundary of the ellipsoids, increases the volume of the leaf.
} 
constant and $\lambda \geq 2 \mu$.

(4) If the restriction $\left.\omega\right|_{\partial M}$ has no singularities in $\partial M$, then $\left.\omega\right|_{\partial M}$ has analogous properties (1)-(3) with respect to the $g_{\lambda, \mu}$-induced metric on the boundary.

These claims should be compared with [HL], Theorem 2.3, Theorem 3.3 and Theorem 7.7, where geometric and homological tautness are shown to be equivalent.

It is interesting to observe that the cycle $F$, not only realizes the minimal volume in its (relative) homology class, but does it for the whole affine hyperplane $[\Omega] \star[\Sigma]=[\Omega] \star[F]$ of relative cycles in $H_{n-1}(M, \partial M ; \mathbf{R})$.

Example. Figure 1a below shows a map $f_{\omega}$ from a surface $M$ with boundary onto an oriented circle $S^{1}$. The map has two Morse-type hyperbolic singularities located on the handle that joins the upper torus (with the circular cut) with the lower torus. The fibers of $f_{\omega}$ form a 1-foliation with two singularities in the interior of $M$. The closed 1-form $\omega$ is defined to be the pull-back $f_{\omega}^{*}(d \theta)$ of the standard 1form $d \theta$ on the circle. It satisfies properties (i) and (ii) from Definition 0.2. Claims (2) and (5) from Theorem A imply that $\omega$ and its restriction to the boundary $\partial M$ are intrinsically harmonic (equivalently, the map $f_{\omega}$ is intrinsically harmonic).

In contrast, the map $f_{\omega}$ and the form $f_{\omega}^{*}(d \theta)$ in Figure $1 \mathrm{c}$ are not intrinsically harmonic. Note that any $\omega$-positive path through any point on the gorge of the slanted handle will be trapped in the upper torus. Thus, (i) from Definition 0.2 does not hold, although (ii) is valid. As in $[\mathrm{Ca}]$, one can show that this failure prevents $\omega$ from being harmonic.

Figure $1 \mathrm{~b}$ depicts the case when Definition 0.2 is also violated, but a weaker Definition 3.1 is satisfied. It follows from Theorem C (Section 3) that the appropriate form $\omega$ is intrinsically harmonic, although its restriction on the boundary $\partial M$ is not.

As shown in [FKL] and [Ho], property (i) from Definition 0.2 can be reformulated in terms of an oriented finite graph $\Gamma_{\omega}(M)$ defined in terms of $M, \omega$. We call it the Calabi graph of $\omega$. When all the leaves of $\mathcal{F}_{\omega}$ are compact, the points of $\Gamma_{\omega}(M)$ are just the connected components of the leaves. It turns out that property (i) from Definition 0.2 holds, if and only if, a similar property is valid for the oriented graph $\Gamma_{\omega}(M)$ : in other words, through any point of the graph there exists an $\omega$-positive loop. Figure 2 below shows the Calabi graphs $\Gamma_{\omega}(M), \Gamma_{\omega}(\partial M)$ of the $\omega$ 's, produced with the help of Figures 1a, 1b and 1c.

Note that claims (1)-(5) from Theorem A are valid regardless of the existence in $\mathcal{F}_{\omega}$ of a non-singular compact leaf $F$, while claims (1)-(4) from Theorem B depend on its existence. We do not know under what most general conditions on $\omega$ the foliation $\mathcal{F}_{\omega}$ has a compact leaf and when such a leaf is non-singular. However, in a few special cases its existence can be guaranteed. For example, if the rank of $\omega$ is one, (i.e. the form has all its periods $\mathbf{R}$-proportional to some rational 


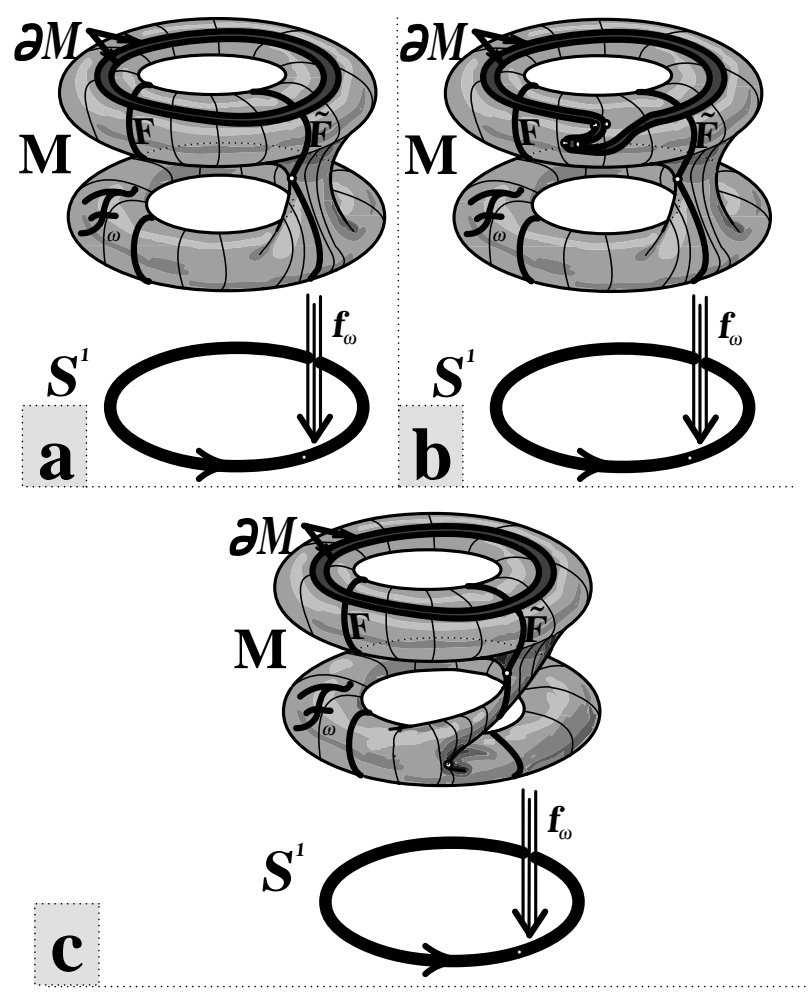

Figure 1

numbers), then all the $\mathcal{F}_{\omega}$-leaves are compact. This case is depicted in Figure 1.

On the other end of the spectrum, if $\omega$ is completely irrational ${ }^{4}$ and Calabi, then on a closed $M$ no leaf components are compact ([FKL]). In fact, a generic (cf. $[\mathrm{FKL}]) \omega$ with all non-compact leaves satisfies the Calabi property ([FKL]). Here a form is called generic if each leaf has at most one singular point.

It also follows from $([\mathrm{FKL}])$ that $\omega$ with no singularities of indices 1 and $(n-1)$ is a Calabi form. Hence,

Corollary 1.1. Statements (1)-(5) of Theorem A are valid for any generic closed 1 -form $\omega$, provided that all the leaves of the foliation $\mathcal{F}_{\omega}$ on $M$ and the ones of $\left.\mathcal{F}_{\omega}\right|_{\partial M}$ on $\partial M$ are non-compact. Also these statements hold if a closed Morse-type form $\omega$ on an $n$-manifold $M$ has no singularities of indices $0,1, n-1, n$ and its

\footnotetext{
${ }^{4}$ i.e. the first Betti number equals to the rank of the subgroup in $\mathbf{R}$, generated over $\mathbf{Q}$ by the $\omega$-periods.
} 


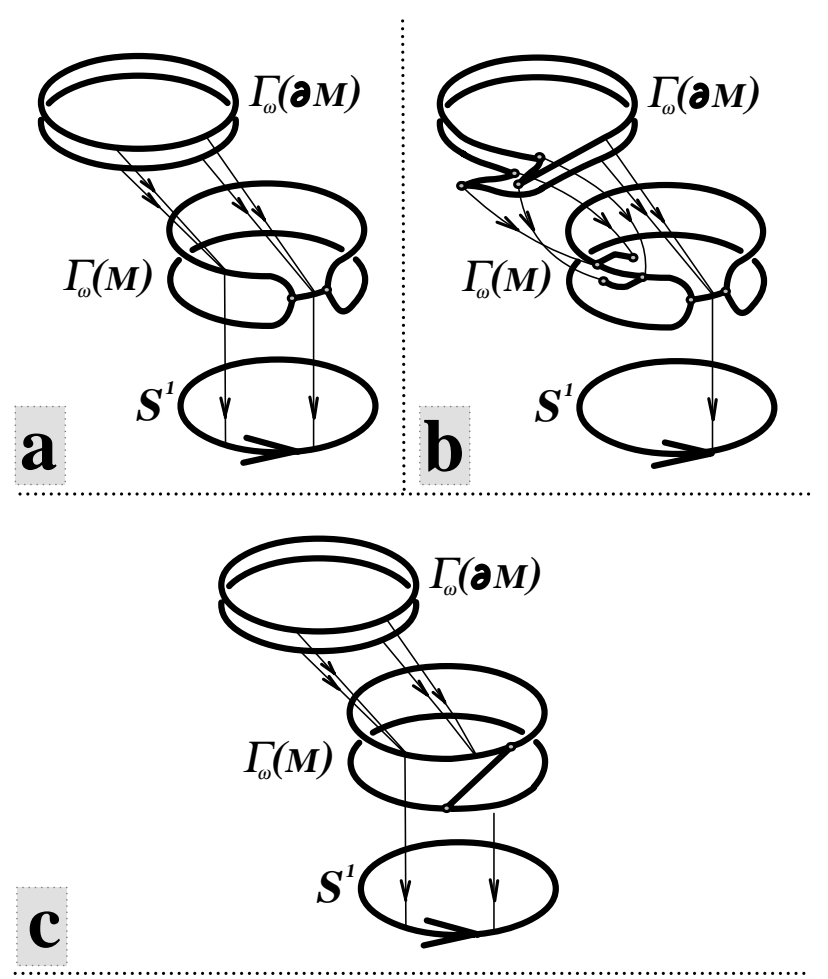

Figure 2

restriction $\omega_{\partial M}-$ no singularities of indices $0,1, n-2, n-1$.

For a Calabi form $\omega$, there are pure homological obstructions to the existence of a compact leaf of $\mathcal{F}_{\omega}$. For instance, if such a leaf exists, there is a non-zero integral element $\theta \in H^{1}(M ; \mathbf{Z})$, such that $\theta \cup[\omega]=0$ in $H^{2}(M ; \mathbf{R})$ ([FKL], Prop. 3). Here $M$ is assumed to be closed.

Therefore, statements (1)-(4) of Theorem B rely on a quite delicate, nongeneric phenomenon.

We say that $\omega$ is homologically $S$-generic, if there is a neighborhood $U$ of the singular set $S_{\omega}^{\star}$, such that, for any compact leaf component of $\mathcal{F}_{\omega}$ which intersects with $U$, there exists a homologous compact leaf which misses $U$. For example, all forms in Figure 1 are $S$-generic. Of course, if all the leaves of $\mathcal{F}_{\omega}$ are compact, $\omega$ is homologically $S$-generic.

Corollary 1.2. Under the hypothesis of Theorem A, for any homologically $S$ - 
generic $\omega$, there exists a metric $g$ on $M$ with the following property:

Any union $F$ of compact $\mathcal{F}_{\omega}$-leaves, which minimizes the $(n-1)$-volume among all the unions $\tilde{F}$ of compact leaf components homologous to $F$ relative $\partial M$, is the volume-minimizing cycle in its relative homology class $[F]$. Moreover, for a given $F$, by a choice of $g$, the volume variation among such $\tilde{F}$ 's can be made arbitrary small.

Proof. Consider one of the metrics $g_{\lambda, \mu}$ whose existence is claimed in Theorem A. Let $F$ be a union of compact leaves, minimizing the $g_{\lambda, \mu}$-induced $(n-1)$-volume among all such unions homologous to $F$. If $F$ misses the $\mu$-ellipsoids, by (1) and (3) from Theorem B, we are done.

Since $\omega$ is homologically $S$-generic, there exists $\mu$, so that any finite collection of compact leaf components is homologous to a collection of compact leaves which miss the $\mu$-ellipsoids. Pick this particular $\mu$ to complete the argument.

Remark. Figure 1 does not suggest that the length variation of all leaves $f_{\omega}^{-1}(\theta)$ in a given relative homology class is very small. By picking the two tori symmetric with respect to rotations around a vertical axis and the tube that joins them tiny, one can get a more accurate illustration of Corollary 1.2.

Corollary 1.3. Let $M, \omega$ be as in Theorem A. Given any finite union $F$ of compact non-singular leaves of $\mathcal{F}_{\omega}$, there exists a metric $g$, so that $\omega$ is harmonic and $F$ is the volume-minimizing cycle in its rel- $-\partial M$-homology class.

Proof. Choose $\mu$ so that $F \subset M_{\omega, \mu}^{\star}$ and apply statements (2) from Theorem A and (1)-(3) from Theorem B.

Remark. In the case depicted in Figure 1a, Corollary 1.3 says that one can "shrink" to the minimum the volume of any preferred non-singular fiber $F$, and still keep the harmonicity of the map $f_{\omega}$.

Corollary 1.4. In the appropriate metric $g$ on $M$, any non-trivial element $[F] \in H_{n-1}(M, \partial M ; \mathbf{Z})$, can be realized as the volume-minimizing fiber of a harmonic Morse-type map $f: M \rightarrow S^{1}$ into the circle. By choice of $g$, the volume variation among the $f$-fibers can be made arbitrary small. Moreover, if $n \geq 6$ and $\pi_{1}(M)=\mathbf{Z}$, one can pick the map $f$ with the minimal in its homotopy class (equivalently, minimal in the homology class $[F]$ ) numbers of singularities of each index.

Proof. Remind that there is a nice correspondence between closed 1-forms $\omega$ with rational periods and smooth maps $f$ into the circle ([Ti]). The correspondence is produced via integration: $f(x)=\int_{\gamma_{x}} \omega \bmod \tau \cdot \mathbf{R}$, where $\tau$ is the least common denominator of the $\omega$-periods and $\gamma_{x}$ denotes a path in $M$, connecting the 
base point with a generic point $x$. Furthermore, cohomologous forms give rise to homotopic maps; harmonic forms produce harmonic maps (for a comprehensive discussion of harmonic maps see [EL]).

First, it is possible to realize the Poincaré-dual of $[F]$ by a closed rational 1form with no singularities of indices 0 and $n$. Then, if $n \geq 6$, by [F], one can find a cohomologous form $\omega^{\prime}$ with the minimal number of singular points of each of the indices $1 \leq i \leq n-1$. In any case, by [FKL] and [Ho], one can deform $\omega^{\prime}$ to a Calabi form $\omega^{\prime \prime}$ with the same distribution of singularities as $\omega^{\prime}$ has. Now apply Theorems A and B to the Calabi form $\omega^{\prime \prime}$.

Remark. Intrinsically harmonic map in Figure 1a has the minimal number of singularities in its homotopy class.

Any closed $(n-1)$-form $\Omega$ on the complement to its singular set gives rise to a 1-dimensional foliation $\mathcal{F}_{\Omega}$. The foliation is tangent to the 1-dimensional kernels of the form $\Omega$ (see $(2.6)$ ). In many cases it is possible to extend $\mathcal{F}_{\Omega}$ across the $\Omega$ singular set. The extension, also denoted by $\mathcal{F}_{\Omega}$, is a 1 -foliation with singularities. In this paper the singularities of $\mathcal{F}_{\Omega}$ will be modeled after the singularities of the gradient flows of Morse functions.

Let $\mathcal{V}=\mathcal{V}_{g}$ denote the $g$-induced volume form on $M$. For a 1 -form $\omega$, its gradient vector field $X_{\omega}$ is defined by the formula: $\left.X_{\omega}\right\rfloor \mathcal{V}=*_{g}(\omega)$. Here "」" denotes the contraction of a differential form by a vector field.

The following proposition is very similar to Theorem A. Although most of the statements of Theorem $\mathrm{A}^{\perp}$ below are, so to speak, the Poincaré-duals of the corresponding statements in Theorem A, due to initial setting (cf. Definition 0.2), there is a subtle break in the duality: the roles of $\omega$ and $\Omega$ are not quite equivalent $-\omega$ is given, while $\Omega$ is produced in a way that is far from being canonic. For example, $\omega$ in Theorem A is not $X_{\omega}$-invariant.

Theorem $\mathbf{A}^{\perp}$. Let the manifold $M$, the 1-form $\omega$, the 2-parametric family of Riemannian metrics $g_{\lambda, \mu}$ and the $(n-1)$-form $\Omega=*_{g_{\lambda, \mu}}(\omega)$ be as in Theorem $A$. Then the following is valid:

(1) The integral curves of the gradient vector field $X_{\omega}=X_{\omega}(\lambda, \mu)$ give rise to a singular ( $\lambda \mu$-independent) 1-foliation $\mathcal{F}_{\Omega}$. Foliations $\mathcal{F}_{\Omega}$ and $\mathcal{F}_{\omega}$ are $g_{\lambda, \mu^{-}}$ orthogonal.

(2) The $X_{\omega}$-generated flow is the $g_{\lambda, \mu}-$ volume-preserving. The form $\Omega$ is invariant under the flow. Hence, $\Omega$ defines an $(n-1)$-dimensional smooth invariant measure, transversal to the foliation $\mathcal{F}_{\Omega}$, - in terms of $([\mathrm{S}])$ a foliation 1-cycle which is not homologous to zero.

(3) The leaves of $\mathcal{F}_{\Omega}$, outside of the $\mu$-ellipsoids, are geodesics in the metric $g_{\lambda, \mu}$. 
(4) If $\left.\omega\right|_{\partial M}$ is non-singular, then, with respect to the $g_{\lambda, \mu}$-induced metric, the analogous statements hold the pair $\left(\partial M,\left.\omega\right|_{\partial M}\right)$.

Theorem $\mathbf{B}^{\perp}$. Under the assumptions and notations of Theorem $A$, the following statements are valid:

(1) Let $L$ be any finite collection of compact and closed $\mathcal{F}_{\Omega}$-leaves (equivalently, of closed trajectories of $X_{\omega}$ ) that does not intersect the $\mu$-ellipsoids around the $\omega$-singularities. Then $L$ minimizes the $g_{\lambda, \mu}$-induced length $l(L)$ among all the 1 cycles $\Gamma$ in $M$, subject to the homological condition $\int_{\Gamma} \omega=\int_{L} \omega$. In particular, the closed geodesic $L$ is of minimal length in its homology class. This minimal length $l(L)=\lambda^{-1} \int_{L} \omega$.

(2) Any 1-cycle $\Gamma$ of the length $l(L)$ and such that $\int_{\Gamma} \omega=\int_{L} \omega$, outside of the $\mu$-ellipsoids, is comprised of a number of compact leaves of $\mathcal{F}_{\Omega}$, restricted to the exterior of the ellipsoids (i.e. $\Gamma$ is comprised of geodesic loops and arcs). The length of the portion $\Gamma_{\mu}^{S}$ of $\Gamma$, lying inside of the $\mu$-ellipsoids, is given by the integral $\lambda^{-1} \int_{\Gamma_{\mu}^{S}} \omega$. When $\lambda \rightarrow+\infty$, it declines as $\sim \lambda^{-1}$; when $\mu \rightarrow 0$, it declines as $\sim \mu$.

(3) For any finite union $\tilde{L}$ of $\mathcal{F}_{\Omega}$-leaves comprised of closed loops and homologous to $L$, the length variation $|l(\tilde{L})-l(L)| \leq K \cdot \mu$, where $K$ is a positive, $\lambda \mu$-independent constant and $\lambda \geq 2 \mu$.

(4) If $\left.\omega\right|_{\partial M}$ is non-singular, then, with respect to the $g_{\lambda, \mu}$-induced metric, the analogous statements hold for the pair $\left(\partial M,\left.\omega\right|_{\partial M}\right)$.

Statement (2) above should be compared with [S], Th. II.20, Th. I.13 and with Th. from $[\mathrm{S} 1]$.

Corollary 1.5. Let $M, \omega$, be as in Theorem $A$ and let $n>2$. Given any finite collection of $\omega$-positive loops $\left\{\gamma_{r}\right\}$, there exists a metric $g$, so that $\omega$ is harmonic and the $\gamma_{r}$ 's are closed, length-minimizing geodesics in their homology classes. Furthermore, their lengths $l\left(\gamma_{r}\right)$ are given by $\int_{\gamma_{r}} \omega$ and, thus, are integral combinations of the $\omega$-periods.

Proof. The proof of this corollary will be embedded in the proofs of Theorems A, $\mathrm{B}$ and $\mathrm{A}^{\perp}, \mathrm{B}^{\perp}$ presented in Section 2.

The proposition below describes some of the asymptotic behavior of the metric spaces $\left(M, g_{\lambda, \mu}\right)$ as $\lambda \rightarrow+\infty$, or as $\mu \rightarrow 0$, (or, in general, as $\lambda, \mu$ approach the virtual boundary of the domain $\left.\left\{\lambda>\max _{k}\left|a_{1}(k)\right|>0, \lambda \geq \mu>0\right\}\right)$. Notice that, for a given $\lambda$, when $\mu \rightarrow 0$, the metric $g_{\lambda, \mu}$ develops infinite singularities around the points of $S_{\omega}$. More specifically, in narrow $2 \mu$-shells surrounding the singularities, an accumulation of curvature takes place; as $\mu \rightarrow 0$, the curvature in the shells tends to infinity. 
Proposition 1.6. Under assumptions and notations of Theorem A, the following statements are valid:

(1) The $n$-volume of $M_{\omega, \mu}^{\star}$ - the complement to the $\mu$-ellipsoids around $S_{\omega}^{\star}-i s$ given by the formula $\lambda^{-2} \int_{M_{\omega, \mu}^{\star}} \omega \wedge \Omega$ and declines as $\sim \lambda^{-2}$ when $\lambda \rightarrow+\infty$. At the same time, the combined volume $V_{\mu}$ of the $\mu$-ellipsoids exceeds $c \cdot \mu^{n}$, c being a positive $\lambda \mu$-independent constant. As a result, for a fixed $\mu$, as $\lambda \rightarrow+\infty$, the volume of the $\mu$-ellipsoids approaches the volume of $M$ at the rate $\sim \lambda^{-2}$.

(2) The $(n-1)$-volume $V(F)$ of any $F$ as in $(1)$, Theorem $B$, is given by the formula $\lambda^{-1} \int_{F} \Omega$ and tends to zero as $\sim \lambda^{-1}$ when $\lambda \rightarrow+\infty$. Thus, the ratio $V(M) / V(F)$ declines as $\sim \lambda^{-1}$ when $\lambda \rightarrow+\infty$.

(3) As $\mu \rightarrow 0$, at the complement to the $\omega$-singular set, the point-wise $g_{\lambda, \mu^{-}}$ norms of $\omega$ and $\Omega$ both converge to the constant function $\lambda$. Alternatively, one can interpret $\lambda$ as $\lim _{\mu \rightarrow 0}\{\|\omega\| / V(M)\}$, where $\|\omega\|$ denotes the integral norm.

Also, for any finite union of leaf components $\tilde{F}$ as in (3), Theorem B,

$$
\lim _{\mu \rightarrow 0} V(M)=\lambda^{-2} \int_{M} \omega \wedge \Omega
$$

and

$$
\lim _{\mu \rightarrow 0} V(\tilde{F})=\lambda^{-1} \int_{F} \Omega .
$$

It seems likely that, for a fixed $\mu$, as $\lambda \rightarrow+\infty$, the spaces $\left(M, g_{\lambda, \mu}\right)$ converge, in the Gromov's sense, to a bouquet of a few $n$-spheres and hemispheres (equipped with a non-standard metric). The spheres are indexed by the $\omega$-singular points and the hemispheres - by the $\left.\omega\right|_{\partial M}$-singular points.

\section{Proofs of the main results}

We start proving Theorems A, B, $\mathrm{A}^{\perp}, \mathrm{B}^{\perp}$, as well as Corollary 1.5 and Proposition 1.6 .

\section{Constructing a candidate for $*{ }_{g} \omega$}

Our immediate goal is to construct a closed $(n-1)$-form $\Omega$ on $M$ serving as a candidate for the form $*_{g}(\omega)$, where $g$ is the Riemannian metric to be defined. In building $\Omega$ we follow closely Calabi's approach ([Ca]), modified for the manifolds with boundary and refined for the purposes of this proof.

We say that an $n$-dimensional differential form $\Theta$ on an oriented $n$-manifold $N$ is positive and write " $\Theta>0$ ", if it defines a non-vanishing section of the canonical bundle $\wedge^{n}\left(T^{*} N\right)$ and induces the preferred orientation of the tangent bundle $T N$. 
We seek to insure the positivity condition $\omega \wedge \Omega>0$ everywhere in $M_{\omega}^{\circ}$.

Let $D^{i}$ denote the open $i$-dimensional unit disk and $D_{+}^{i}-$ its half. Let $S^{1}$ be the unit circle.

According to the relative Calabi property of $\omega$, for each $x \in M_{\omega}^{\star}=M \backslash S_{\omega}^{\star}$, there exists an $\omega$-positive loop $\gamma$, moreover, when $x \in \partial M_{\omega}^{\circ}, \gamma$ lies in $\partial M$. If $x$ is not on the boundary, such a $\gamma$ has an open tubular neighborhood $U_{\gamma}$, parameterized by a diffeomorphism

$$
h_{\gamma}: S^{1} \times D^{n-1} \longrightarrow U_{\gamma} \subset M_{\omega}^{\star},
$$

so that each $t$-path $\gamma_{a}(t)=h_{\gamma}(t, a)$ (with $a \in D^{n-1}$ and $t \in S^{1}$ ) is $\omega$-positive. For this we need $M$ to be orientable. The non-orientable $M$ 's can be treated in a similar way (for the appropriate modifications see $([\mathrm{Ca}])$. Similarly, any $\omega-$ positive path $\gamma$ in $\partial M$, has an open tubular neighborhood in $M$, parameterized by a diffeomorphism

$$
h_{\gamma}:\left(S^{1} \times D_{+}^{n-1}, S^{1} \times D^{n-2}\right) \longrightarrow\left(U_{\gamma}, U_{\gamma}^{\partial}\right) \subset(M, \partial M),
$$

so that, for each $a \in D_{+}^{n-1}, \gamma_{a}(t)=h_{\gamma}(t, a)$ is an $\omega$-positive path.

We call such parameterized neighborhoods $U_{\gamma}$ of $\gamma$ " $\omega$-positive tubes".

Lemma 2.1. Let $N$ be a compact $n$-manifold and $\omega$ - a 1-form satisfying the relative Calabi condition (cf. Def. 0.2). Let $\gamma$ be an $\omega$-positive loop in the interior of $N$. Then, for $n>2$, there exists an open regular neighborhood $V$ of $\gamma$, such that $\left.\omega\right|_{N \backslash V}$ also possess the relative Calabi property.

Proof. By definition, through each $x \in N_{\omega}^{\star}$ there exists an $\omega$-positive loop $\gamma_{x}$. For $n>2$ and any point $x \in N_{\omega}^{\star} \backslash \gamma$ the loop $\gamma_{x}$ can be perturbed, so that it misses the loop $\gamma$ and and still is $\omega$-positive. Moreover, one can find an open $\omega$-positive tube $U\left(\gamma_{x}\right)$ around $\gamma_{x}$ which does not intersect $\gamma$. Take $U$ to be an $\omega$-positive tube around $\gamma$. Then $U$ together with the tubes $\left\{U\left(\gamma_{x}\right)\right\}_{x \in N_{\omega}^{\star} \backslash \gamma}$ form an open cover $\mathcal{U}^{\prime}$ of $N_{\omega}^{\star}$. Let $\mathcal{U}$ be the union of elements from $\mathcal{U}^{\prime}$ and the open ellipsoids that are centered on the $\omega$-singular points and do not intersect with $U$. Since $N$ is compact, one can find a finite subcover of $\mathcal{U}$ consisting of $U$, the ellipsoids and finitely many $U\left(\gamma_{x}\right)$ 's. Denote them by $U\left(\gamma_{j}\right)$. Pick an $\omega$-positive tube $V$ around $\gamma$, compactly contained in $U$, which does non intersect the tubes $\left\{U\left(\gamma_{j}\right)\right\}_{j}$. Clearly, on $N \backslash V$, $\omega$ enjoys the relative Calabi property.

Let us fix $\epsilon>0$, such that all the $2 \epsilon$-ellipsoids $\left\{B_{2 \epsilon, j}\right\}_{j},\left\{B_{2 \epsilon, k}^{+}\right\}_{k}$ are disjoint. Consider the compliment $M_{\omega, \epsilon}^{\star}$ to the open $\epsilon$-ellipsoids. For each point $x \in M_{\omega, \epsilon}^{\star}$, pick an $\omega$-positive loop $\gamma_{x}$ surrounded by an $\omega$-positive tube $U_{\gamma_{x}}$. Clearly, as $x$ ranges in $M_{\omega, \epsilon}^{\star},\left\{U_{\gamma_{x}}\right\}_{x}$ form an open cover of $M_{\omega, \epsilon}^{\star}$. Since $M$ is compact, this cover has a finite subcover $\left\{U_{l}\right\}_{l}$. In fact, $\left\{U_{l}\right\}_{l}$ together with $\left\{B_{2 \epsilon, j}\right\}_{j}$ and $\left\{B_{2 \epsilon, k}^{+}\right\}_{k}$ form a finite cover of $M$. This $\omega$-subordinate cover $\mathcal{U}=\mathcal{U}(\epsilon)$, comprised of $\omega$-positive 
tubes, the $2 \epsilon$-ellipsoids and the half-ellipsoids centered on the singularities, will be fixed in the considerations to follow.

We start assembling a closed $(n-1)$-form $\Omega$ from special closed forms with supports in the elements of $\mathcal{U}$.

Since there are only finitely many open sets $U_{l}$ (with their compact closures being contained in $M_{\omega}^{\star}$ ), there exists a positive $\delta=\delta(\epsilon)$, such that each $\omega$-positive tube $U_{l}$ from $\mathcal{U}$ has an empty intersection with the $\delta$-ellipsoids surrounding $S_{\omega}^{\star}$. Figure 3 illustrates a choice of $\delta$ at a typical singularity; the $\omega$-positive tubes surrounding the singularity are shaded.

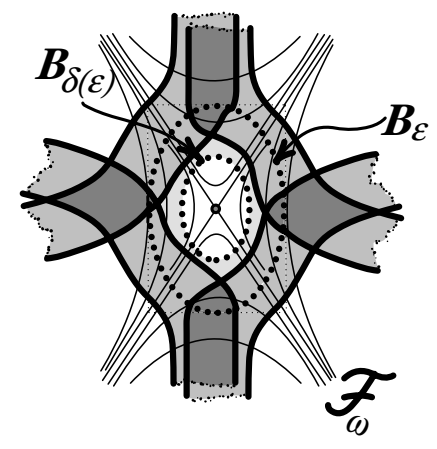

Figure 3

Let $\varphi_{j}: M \rightarrow[0,1]$ be a smooth bell-shaped function with the support in $B_{2 \epsilon, j}$ which is identically 1 in $B_{\epsilon, j} \supset B_{\delta, j}$. We may assume that, in the calibrated Morse coordinates, $\varphi_{j}=\varphi\left(\sum_{i=1}^{n} a_{i}^{2} x_{i}^{2}\right)$, where $\varphi$ is a smooth bell-shaped function in one variable with the derivative $\varphi^{\prime}<2 / \epsilon$ (see Figure 4 ).

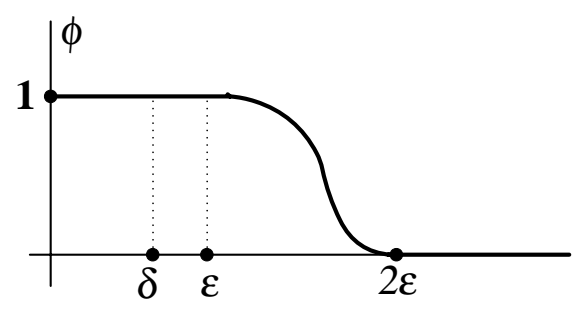

Figure 4

Functions $\varphi_{k}^{+}: M \rightarrow[0,1]$ with support in $B_{2 \epsilon, k}^{+}$and of the form $\varphi\left(\sum_{i=1}^{n} a_{i}^{2} x_{i}^{2}\right)$ can be defined in a similar way. 
Let $g_{E, j}$ and $g_{E, k}$, respectively, denote the Euclidean metric in the Morse coordinates around the singularities $A_{j}$ and $A_{k}^{\partial}$.

In the ellipsoid $B_{2 \epsilon, j}$, put $\Omega_{j}^{\prime}=*_{g_{E, j}}(\omega)$ and, in $B_{2 \epsilon, k}^{+}$, put $\Omega_{k}^{\prime}=*_{g_{E, k}}(\omega)$. Since, due to the calibration of the coordinates, $\omega$ is harmonic with respect to $g_{E, j}$ and $g_{E, k}$, the forms $\Omega_{j}^{\prime}$ and $\Omega_{k}^{\prime}$ are closed. By the Poincaré Lemma, $\Omega_{j}^{\prime}=\mathrm{d} \Psi_{j}$ for a $(n-2)$-form $\Psi_{j}$ in $B_{2 \epsilon, j}$. In fact, in the Morse coordinates $\left\{x_{i}\right\}$, the form $\Psi_{j}$ has coefficients that are quadratic monomials in the $x_{i}$ 's. Analogously, in $B_{2 \epsilon, k}^{+}$, $\Omega_{k}^{\prime}=\mathrm{d} \Psi_{k}$. The coefficients of $\Psi_{k}$ are linear in the $x_{1}$ and quadratic in the rest of the coordinates.

Now let $\Omega_{j}=\mathrm{d}\left(\varphi_{j} \cdot \Psi_{j}\right)$ and $\Omega_{k}^{+}=\mathrm{d}\left(\varphi_{k}^{+} \cdot \Psi_{k}\right)$. Notice that, in $B_{\delta, j}, \Omega_{j}=\Omega_{j}^{\prime}$ and, in $B_{\delta, k}^{+}, \Omega_{k}^{+}=\Omega_{k}^{\prime}$. Evidently, $\Omega_{j}$ and $\Omega_{k}^{+}$are well-defined globally and are closed.

We proceed to construct a closed $(n-1)$-form $\Omega_{l}$ supported in the $\omega$-positive tube $U_{l} \in \mathcal{U}$. Its construction employs the $D^{n-1}$ - or $D_{+}^{n-1}$-bundle structure of the tube $U_{l}$ over the loop $\gamma_{l}$, induced by the diffeomorphism $h_{l}$ in (2.1) or (2.2).

Let $t$ denote the $S^{1}$-coordinate in $S^{1} \times D^{n-1}$ and in $S^{1} \times D_{+}^{n-1}$. Let $u_{1}, u_{2}$, $\ldots, u_{n-1}$ stand for the disk-directed coordinates. In the case of $D_{+}^{n-1}$, let $u_{2}, \ldots$, $u_{n-1}$ be the coordinates along the equator $D^{n-2} \subset D_{+}^{n-1}$ (see Figure 5).

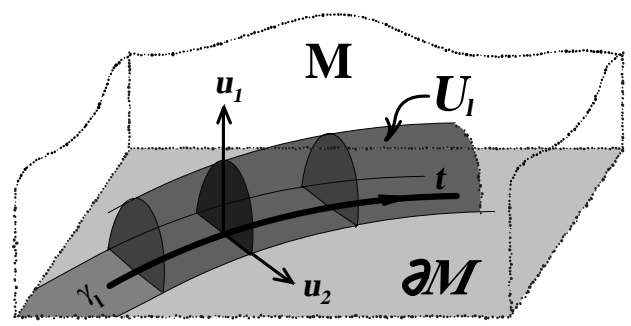

Figure 5

Put $\Theta=d u_{1} \wedge d u_{2} \wedge \cdots \wedge d u_{n-1}$ and use the diffeomorphism $h_{l}^{-1}$ to pull-back the form $\Theta$ to a form $\Omega_{l}^{\prime}$ in the $U_{l}$. This form has the following properties:

(1) $\mathrm{d} \Omega_{l}^{\prime}=0$.

(2) $\omega \wedge \Omega_{l}^{\prime}>0$.

(3) If the core $\gamma_{l}$ of $U_{l}$ lies in $\partial M$, then $\Omega_{l}^{\prime}$, being restricted to $\partial M$, vanishes.

(1) is valid because $d \Theta=0$; (2) follows from the fundamental positivity property $\omega\left(h_{l *}\left(\partial_{t}\right)\right)>0$ of the diffeomorphism $h_{l}$; and $(3)$ - from the observation that $\Theta$, evaluated at the poly-vector $\partial_{t} \wedge \partial_{2} \wedge \cdots \wedge \partial_{n-1}$, vanishes. 
Note that we do not insist that either $d t=h_{l}^{*}(\omega)$, or that the $h_{l}$-images of the $(n-1)$-disks would lie in the leaves of the foliation $\mathcal{F}_{\omega}$. Actually, the latter property is achievable and useful.

Let $\tilde{D}^{n-1} \subset D^{n-1}, \tilde{D}_{+}^{n-1} \subset D_{+}^{n-1}$ be the concentric subdisks of, say, radius $1 / 2$. Denote by $\tilde{\varphi}_{l}$ a smooth function on $\mathbf{R}^{n-1}$, supported in $D^{n-1}$ and being identically 1 in $\tilde{D}^{n-1}$. Let $\varphi_{l}: M \rightarrow[0,1]$ be a smooth function with the support in the tube $U_{l}$ and such that its pull-back $h_{l}^{*}\left(\varphi_{l}\right)$ is $t$-independent. Being restricted at each of the disk fibers in $S^{1} \times D^{n-1}$ or in $S^{1} \times D_{+}^{n-1}$, it coincides with the function $\tilde{\varphi}_{l}$.

Now consider the globally-defined form $\Omega_{l}=\tilde{\varphi}_{l} \cdot \Omega_{l}^{\prime}$ supported in $U_{l}$. Because $h_{l}^{*}\left(\varphi_{l}\right)$ is $t$-independent, $\Omega_{l}$ is a closed form on $M$. By property (2) above and the choice of $\varphi_{l}, \omega \wedge \Omega_{l}>0$ in $U_{l}$. By property (3), $\left.\Omega_{l}\right|_{\partial M}=0$. Hence, the sum $\Omega^{\dagger}=\sum_{l} \Omega_{l}$ satisfies the following list of properties:

(i) Support of $\Omega^{\dagger}$ is contained in the interior of $M_{\omega, \delta}^{\star}$.

(ii) $\omega \wedge \Omega^{\dagger}>0$ in $M_{\omega, \epsilon}^{\star}$.

(iii) $\mathrm{d} \Omega^{\dagger}=0$.

(iv) $\left.\Omega^{\dagger}\right|_{\partial M}=0$.

For some of the applications (cf. Corollary 1.5), it will be useful to choose some of the $\omega$-positive tubes $U_{l}$ with extra-care. In fact, given any finite number of $\omega$ positive loops $\left\{\gamma_{l}\right\}$, by Lemma 2.1 , it is possible to surround them with $\omega$-positive tubes $V_{l}$, so that each $V_{l}$ is compactly contained in the appropriate $U_{l}$ from the finite cover $\mathcal{U}$ and $V_{l} \cap U_{l^{\prime}}=\phi$ for any $l^{\prime}$ distinct from $l$. Moreover, for a given collection $\left\{\gamma_{l}\right\}$, one can pick $\epsilon>0$ and the $V_{l}$ 's in such a way that they have empty intersections with all the $2 \epsilon$-ellipsoids. As a result, the $V_{l}$ 's do not interact with the rest of elements of the cover $\mathcal{U}$.

Let us form a linear combination of closed $(n-1)$-forms

$$
\Omega=\sum_{j} \Omega_{j}+\sum_{k} \Omega_{k}^{+}+q \cdot \Omega^{\dagger},
$$

where the value of positive constant $q$ will be chosen later. The first two sums are comprised of exact $(n-1)$-forms supported in the $2 \epsilon$-ellipsoids around the singular set $S_{\omega}^{\star}$.

Although, for each singularity $A_{j}, \omega \wedge *_{g_{E, j}}(\omega)>0$ everywhere, except at the origin $A_{j}$, a modification of $*_{g_{E, j}}(\omega)$ - the form $\Omega_{j}=\mathrm{d}\left(\varphi_{j} \cdot \Psi_{j}\right)$ - retains this property inside of the ellipsoid $B_{\epsilon, j}$ ( $\varphi_{j}$ is constant there), and $\omega \wedge \mathrm{d}\left(\varphi_{j} \cdot \Psi_{j}\right)$ fails to be positive somewhere in the shell $B_{2 \epsilon, j} \backslash B_{\epsilon, j}$. In a similar way, $\omega \wedge \mathrm{d}\left(\varphi_{k}^{+} \cdot \Psi_{k}\right)$ fails to be positive in the the shell $B_{2 \epsilon, k}^{+} \backslash B_{\epsilon, k}^{+}$. By choosing the constant $q$ in $(2.4)$ large enough, one can compensate for these failures of positivity and ensure that $\omega \wedge \Omega>0$ everywhere in $M_{\omega}^{\star}$. The choice of $q$, in each of the shells, is prescribed by the requirements

$$
\omega \wedge\left[\mathrm{d}\left(\varphi_{j} \cdot \Psi_{j}\right)+q \cdot \Omega^{\dagger}\right]>0
$$




$$
\omega \wedge\left[\mathrm{d}\left(\varphi_{k}^{+} \cdot \Psi_{k}\right)+q \cdot \Omega^{\dagger}\right]>0 .
$$

Due to the choice of $\omega$-positive tubes $\left\{U_{l}\right\}$ which cover the shells, the existence of an appropriate $q$ is clear. With this choice of $q$, the construction of the form $\Omega$ is complete.

Let $B_{\epsilon, k}^{\partial}=B_{\epsilon, k}^{+} \cap \partial M$. In the following lemma we list a few properties of $\Omega$.

Lemma 2.2. Given a Calabi form $\omega$ and $\epsilon>0$, there exists an $(n-1)$-form $\Omega$ and a positive $\delta=\delta(\epsilon)$, so that:

1) $\mathrm{d} \Omega=0$.

2) $\omega \wedge \Omega>0$ in $M_{\omega}^{\circ}$.

3) For each $\omega$-singularity $A_{j}$, there exists an euclidean metric $g_{E, j}$ such that, in the $\delta$-ellipsoid $B_{\delta, j}, \Omega=*_{g_{E, j}}(\omega)$.

4) For each $\left.\omega\right|_{\partial M}$-singularity $A_{k}^{+}$, there exists an euclidean metric $g_{E, k}$ such that, in the $\delta$-half-ellipsoid $B_{\delta, k}^{+}, \Omega=*_{g_{E, k}}(\omega)$.

5) $\left.\Omega\right|_{\partial M_{\omega, 2 \epsilon}^{\circ}}=0$. In the Morse coordinates, where $\omega=a_{1} d x_{1}+\sum_{i=2}^{n} a_{i} x_{i} d x_{i}$ and $\varphi_{k}^{+}=\varphi_{k}^{+}\left(0, x_{2}, \ldots, x_{n}\right)$, one has: $\left.\Omega\right|_{B_{2 \epsilon, k}^{\partial}}=a_{1} \cdot \mathrm{d}\left(\varphi_{k}^{+} \cdot x_{2} d x_{3} \wedge \cdots \wedge d x_{n}\right)$ and $\left.\Omega\right|_{B_{\delta, k}^{\partial}}=a_{1} \cdot d x_{2} \wedge \cdots \wedge d x_{n}$.

6) For any given finite set of $\omega$-positive loops $\left\{\gamma_{l}\right\}$, one can find $\omega$-positive tubes $V_{l} \supset \gamma_{l}$, in which $\Omega=\left(h_{l}^{-1}\right)^{*}\left(d u_{1} \wedge \cdots \wedge d u_{n-1}\right)$, the diffeomorphism $h_{l}$ being defined as in (2.1).

Proof. All the statements of the lemma, except 5), have been established in the preceding arguments. Property 5) is a direct implication of property 4).

Proposition 2.3. For a form $\omega$ which satisfies the relative Calabi property, any compact leaf component $F$ of the foliation $\mathcal{F}_{\omega}$ defines a non-trivial element in the homology $H_{n-1}(M, \partial M ; \mathbf{R})$.

Proof. Suppose that the cycle $(F, \partial F)$ actually, is homologous (cobordant) to zero in $(M, \partial M)$. Let $(W, \partial W)$ be the appropriate null-cobordism with $\partial W=F \cup_{\partial F} G$. Here $G \subset \partial W$ is the portion of the boundary contained in $\partial M$ and $\partial F=\partial G$.

Without loss of generality, we may assume that $\partial F \subset \partial M_{\omega, 2 \epsilon}^{\circ}$ : indeed, if $\partial F \subset \partial M_{\omega}^{\circ}$, then one can find $\epsilon$ small enough, so that $\partial F$ does not intersect the $2 \epsilon$-ellipsoids centered at points of $S_{\omega}^{\partial}$. For this choice of $\epsilon$, construct $\Omega$ as in Lemma 2.2 .

If a non-singular $F$ is such that $\partial F \cap S_{\omega}^{\partial} \neq \phi$, then there exists a near-by homologous leaf $F^{\prime}$ which does not intersect $S_{\omega}^{\partial}$ and the following argument applies to $F=F^{\prime}$.

Since $\mathrm{d} \Omega=0$, by the Stokes' Theorem, we get $\int_{F} \Omega=\int_{G} \Omega$. Because $\omega \wedge \Omega>0$ in $M_{\omega}^{\circ},\left.\Omega\right|_{F}>0$ and the left-hand side of the previous equality is strictly positive. At the same time, since $\left.\Omega\right|_{\partial M}=\mathrm{d}(\varphi \cdot \Psi)$ for an $(n-2)$-form $\Psi$, 
and since the function $\varphi$ is supported in the $2 \epsilon$-ellipsoids, $\int_{G} \Omega=\int_{\partial G} \varphi \cdot \Psi=0$. The contradiction proves the proposition.

Now, for a given Calabi 1-form $\omega$, we would like to make a few observations concerning the freedom to choose a closed $(n-1)$-form $\Omega$ with the properties described in Theorem A and Lemma 2.2. In particular, we are concerned with the degrees of freedom to choose the periods of $\Omega$. An interesting related question is: "For a given form $\omega$, is it possible to construct $\Omega$ with rational (integral) periods?" We do not know how to tackle this problem, in a sense, a problem of "quantization" of the appropriate $* g$-operator. However, once $\Omega$ has been constructed, it is possible to change its periods in a controlled and discrete way. Of course, it is always possible to replace $\Omega$ with $\Omega^{\prime}=r \Omega$, where $r$ is a positive real number.

Consider the cone of cycles in $H_{1}(M ; \mathbf{Z})$ represented by finite unions of $\omega$ positive loops. Denote this set by $H_{\omega}^{+}$. The set $H_{\omega}^{+}$is closed under the $\mathbf{Z}_{+^{-}}$ linear combinations of its elements: disjoint unions of $\omega$-positive loops realize the appropriate operations in $H_{1}(M ; \mathbf{Z})$.

If $\omega$ has a positive period along a loop $\gamma$, then there exists a function $f$ on $M$ with support in a small neighborhood of $\gamma$ and such that $\gamma$ is $(\omega+d f)$-positive. Hence, given a disjoint union of loops $\gamma_{r}$, subject to the condition $\int_{\gamma_{r}} \omega>0$, there is a representative $\omega^{\prime}$ in the cohomology class of $\omega$, so that all the $\gamma_{r}$ 's become $\omega^{\prime}-$ positive. Consider elements $[\gamma]$ of $H_{1}(M ; \mathbf{Z})$ which satisfy the positivity condition $[\omega] \star[\gamma]>0$. They form a cone (a semi-group) $\mathcal{H}_{[\omega]}^{+}$containing $H_{\omega}^{+}$. One can pick a basis of $H_{1}(M ; \mathbf{Z})$ lying in $\mathcal{H}_{[\omega]}^{+}$and represented by a few loops $\gamma_{r}$ ( $r$ ranges from 1 to the first Betti number of $M$ ). Using the previous remarks, there exists a closed form $\omega^{\prime}$ cohomologous to $\omega$, for which $H_{\omega^{\prime}}^{+}$contains the $\mathbf{Z}_{+}$-cone spanned by the basic elements $\left\{\gamma_{r}\right\}$. Therefore, as $\omega$ ranges in its cohomology class, the cones $H_{\omega}^{+}$ change and include positive cones as large as the "half-space" $\mathcal{H}_{[\omega]}^{+}$, spanned by a bases of $H_{1}(M ; \mathbf{Z})$. Of course, for some $\omega$ 's, $H_{\omega}^{+}$can be very small, even empty (this can not happen for Calabi forms).

The natural intersection pairing $\circ: H_{1}(M ; \mathbf{Z}) \otimes_{\mathbf{Z}} H_{n-1}(M, \partial M ; \mathbf{Z}) \rightarrow \mathbf{Z}$ restricts to the pairing

$$
\circ: H_{\omega}^{+} \otimes \mathbf{Z}_{+} H_{n-1}(M, \partial M ; \mathbf{Z}) \rightarrow \mathbf{Z} .
$$

Let us fix a basis $\left\{\Sigma_{1}, \ldots, \Sigma_{r}\right\}$ in the torsion-free group $H_{n-1}(M, \partial M ; \mathbf{Z})$. One can realize $\Sigma_{k}$ 's by embedded smooth hypersurfaces.

Proposition 2.4. Given a positive number $s$ and a closed $(n-1)$-form $\Omega$ with the properties 1), 2) and 5) of Lemma 2.2 (equivalently, with the properties described in Theorem A) and an element $\gamma \in H_{\omega}^{+}$, one can change $\Omega$ to a new form $\Omega^{\prime}$, still possessing the same properties and such that

$$
\int_{\Sigma_{k}} \Omega^{\prime}=\int_{\Sigma_{k}} \Omega+s\left(\gamma \circ \Sigma_{k}\right), \quad 1 \leq k \leq r .
$$


Also one can replace $\Omega$ with $q \cdot \Omega(q>0)$, while preserving its desired properties.

Proof. By general position argument, one can assume that all the $\omega$-positive loops $\left\{\gamma_{l}\right\}$, comprising $\gamma$, are transversal to all the hypersurfaces $\left\{\Sigma_{k}\right\}$. Moreover, the $\gamma_{l}$ 's can be surrounded by $\omega$-positive tubes $h_{l}: S^{1} \times D^{n-1} \rightarrow U_{l} \subset M$, so that each $t$-loop $h_{l}(\sim, a), a \in D^{n-1}$, is also transversal to the $\Sigma_{k}$ 's. In fact, the trivialization $h_{l}^{-1}$ of the tube $U_{l}$ can be chosen so that $U_{l} \cap \Sigma_{k}=\bigcup h_{l}\left(t_{k_{j}} \times D^{n-1}\right)$ for all the $k$ 's and some $t_{k_{j}} \in S^{1}$. Furthermore, the tubes $\left\{U_{l}\right\}$ can be constructed thin enough in order for the disks $\left(U_{l} \cap \Sigma_{k}\right)_{j}=h_{l}\left(t_{k_{j}} \times D^{n-1}\right)$ to be disjoint.

With this choice of the trivializations, one can construct a $t$-independent (and thus closed) $(n-1)$-form $\Omega_{l}$ which is supported in $U_{l}$ and such that, for each intersection point (i.e. for each triple $(l, k, j)$ ),

$$
\int_{\left(U_{l} \cap \Sigma_{k}\right)_{j}} \Omega_{l}=\int_{D^{n-1}} h_{l}^{*}\left(\Omega_{l}\right)= \pm s .
$$

The sign here equals to the orientations-induced sign of the intersection of $\gamma_{l}$ with $\Sigma_{k}$ at the appropriate point. Clearly, replacing the original $\Omega$ with $\Omega+\Omega_{l}$ only reinforces the positivity property $\omega \wedge \Omega>0$ in $M_{\omega}^{\circ}$. Of course, this modification of $\Omega$ calls for a change of the related metrics $g_{\lambda, \mu}$ in Theorem A.

Example. Consider the map and the form shown in Figure 1a. The free abelian group $H_{1}(M ; \mathbf{Z})$ of rank 3 is generated by the meridian $a$ and the longitude $b$ of the lower "torus" together with the longitude $c$ of the upper "torus". As the picture suggests, $b$ and $c$ can be chosen to be $\omega$-positive. Moreover, one can see that, for any integer $n$, the class $b+n a$ is also realizable by an $\omega$-positive loop. In order to construct such a representative, one follows $b$ in the positive direction until it reaches the intersection point with $a$. Just before the intersection, one adds a short $\omega$-positive spiral $n a$ to $b$ (as in the Dehn's twist). Thus, any integral combination $p b+q c+n a$ with $p \geq 0, q \geq 0$ and such that $n \neq 0$ implies $p>0$, belongs to the cone $H_{\omega}^{+}$. In fact, with a bit more effort one can check that $H_{\omega}^{+}$is comprised of such elements. The set $\mathcal{H}_{[\omega]}^{+}$is defined by the inequality $\{p b+q c+n a \mid p+q>0\}$ (with $p, q$ not necessarily positive) and evidently, contains $H_{\omega}^{+}$.

The group $H_{1}(M, \partial M ; \mathbf{Z})$ of rank 3 is generated by the loops $a, b$ and an arc $d$, Poincaré-dual to the loop $c$. The intersections of these generators with a typical $\omega$-positive loop $\gamma=p b+q c+n a$ are: $\gamma \circ a=p, \gamma \circ b=-n, \gamma \circ a=q$.

According to Proposition 2.4, the vector of the $(a, b, d)$-periods of $\Omega$ can be changed by adding to it any vector of the form $s(p,-n, q), s$ being a positive real number. (Recall that here $p=0$ calls for $n=0$ ).

Borrowing arguments from the proof of Proposition 2.4, one can establish the following proposition, whose proof we postpone until the end of the next subsection. One can think of this proposition as a "taut" version of the rational Poincaré duality (in dimensions 1 and $n-1$ ). 
Proposition 2.5. Let $M$ and $\omega$ be as in Theorem $A$ and $\left\{\gamma_{l}\right\}$ - any finite collection of $\omega$-positive loops. Assume that $n=\operatorname{dim} M>2$. Then there exists a Riemannian metric on $M$, such that:

(1) $\omega$ is harmonic,

(2) $\left\{\gamma_{l}\right\}$ are geodesics; furthermore, each $\gamma_{l}$ is a core of an $\omega$-positive tube $U_{l}$ which itself consists of geodesic loops $\gamma_{l}^{\prime}$ of a fixed length (equal to $\int_{\gamma_{l}} \omega$ ),

(3) any hypersurface $(\Sigma, \partial \Sigma) \subset(M . \partial M)$, which minimizes the volume in its relative integral homology class, has only positive intersections with some of (actually, a big majority of) the geodesic loops $\gamma_{l}^{\prime}$. As a result, the cardinality of $\Sigma \cap \gamma_{l}^{\prime}$ equals $\left|\Sigma \circ \gamma_{l}^{\prime}\right|$.

In fact, for any non-zero element $[\omega] \in H^{n-1}(M ; \mathbf{R})$, there exists a representative closed 1-form $\omega$, a set of $\omega$-positive loops $\left\{\gamma_{l}\right\}$ which form a basis in $H_{1}(M ; \mathbf{Z}) /$ Tor , and a metric on $M$, so that statements (1)-(3) above are valid. In particular, there exists a metric in which a basis of $H_{n-1}(M, \partial M ; \mathbf{Z})$ is realized by volume minimizing relative cycles (hypersurfaces with singularities) $\left\{\Sigma_{k}\right\}$, a basis in $H_{1}(M ; \mathbf{Z}) /$ Tor - by geodesic loops and their mutual intersections have the minimal cardinality prescribed by the homology intersection $\left[\Sigma_{k}\right] \circ\left[\gamma_{l}\right]$.

\section{Constructing the family of metrics $g_{\lambda, \mu}$}

Our next goal is to produce a Riemannian metric $g$ on $M$, or rather a twoparametric family of metrics $g_{\lambda, \mu}$ (see Theorem A), so that $\Omega={ }_{g} \omega$.

An 1 -form $\omega$, in the complement to its singular set $S_{\omega}$, defines an $(n-1)$ dimensional distribution $K_{\omega}$ :

$$
K_{\omega, x}=\left\{v \in T_{x} M \mid \omega(v)=0\right\} .
$$

If $\omega$ is closed, $K_{\omega}$ is integrable and formed by hyperplanes, tangent to the foliation $\mathcal{F}_{\omega}$.

An $(n-1)$-form $\Omega$, in the complement to its singular set $S_{\Omega}$, defines a 1 dimensional distribution $K_{\Omega}$ :

$$
K_{\Omega, x}=\left\{v \in T_{x} M \mid \quad \Omega(v \wedge w)=0 \text { for all } w \in \wedge^{n-2}\left(T_{x} M\right)\right\}
$$

Being 1-dimensional, $K_{\Omega}$ is integrable as well.

If, at $x \in M$, the form $(\omega \wedge \Omega)_{x}>0$, the subspaces $K_{\omega, x}$ and $K_{\Omega, x}$ are transversal.

Let us consider the restrictions $\Omega_{\omega}=\left.\Omega\right|_{K_{\omega}}$ and $\omega_{\Omega}=\left.\omega\right|_{K_{\Omega}}$. If $(\omega \wedge \Omega)_{x}>0$, then $\Omega_{\omega, x} \neq 0$ and $\omega_{\Omega, x} \neq 0$.

Given a Riemannian metric $g$ on an $n$-dimensional $M$, denote by $\mathcal{V}^{n}=\mathcal{V}^{n}(g)$ its volume form, by $\mathcal{V}_{\omega}^{n-1}=\mathcal{V}_{\omega}^{n-1}(g)$ - the $g$-induced $(n-1)$-volume form on the bundle $K_{\omega}$ and by $\mathcal{V}_{\Omega}^{1}=\mathcal{V}_{\Omega}^{1}(g)$ - the $g$-induced 1 -volume form on the bundle $K_{\Omega}$.

We shall compare $\Omega_{\omega}$ with $\mathcal{V}_{\omega}^{n-1}$ and $\omega_{\Omega}$ with $\mathcal{V}_{\Omega}^{1}$. This comparison will depend on a function $h: M \rightarrow \mathbf{R}_{+}$which will be constructed prior to the construction of 
the desired metric $g$. In turn, the choice of $h$ will depend essentially on two real positive parameters $\lambda, \mu$. In what follows, we assume that $2 \mu<\delta(\epsilon)$, where $\epsilon, \delta(\epsilon)$ have been chosen in the process of constructing the $\omega$-subordinate cover $\mathcal{U}$.

Here is a list of properties that characterize $h$ and are illustrated in Figure 6:

(1) $h$ is smooth in $M_{\omega}^{\circ}$ and its square $h^{2}-$ in $M$.

(2) On the set $M_{\omega, 2 \mu}^{\star}$ the function $h$ is identically $\lambda$.

(3) In each of the ellipsoids $B_{\mu, j}, h$ coincides with the norm-function

$$
\|\omega\|_{E, j}=\left(\sum_{i=1}^{n} a_{i}^{2} x_{i}^{2}\right)^{1 / 2}
$$

in the euclidean metric $g_{E, j}$.

(4) In each of the half-ellipsoids $B_{\mu, k}^{+}, h$ coincides with the norm-function

$$
\|\omega\|_{E, k}=\left(a_{1}^{2}+\sum_{i=2}^{n} a_{i}^{2} x_{i}^{2}\right)^{1 / 2}
$$

in the euclidean metric $g_{E, k}$.
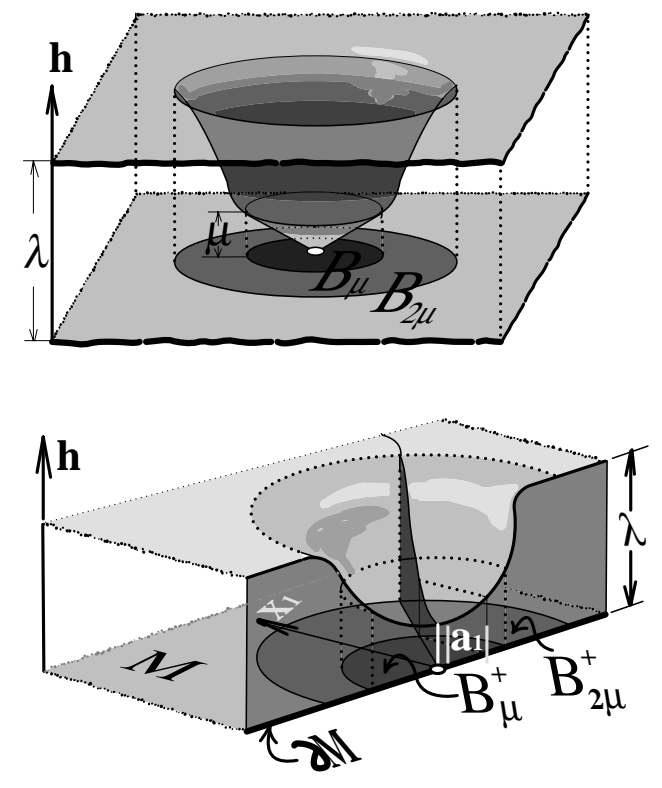

Figure 6

Lemma 2.5. Let $h: M \rightarrow \mathbf{R}_{+}$be as in (2.7). For any 1 -form $\omega$ and an $(n-1)$ form $\Omega$, so that 
1) $\omega \wedge \Omega>0$ in $M_{\omega}^{\circ}$ and

2) $\Omega=*_{g_{E}}(\omega)$ in the $\mu$-ellipsoids around $S_{\omega}^{\star}$, there exists a Riemannian metric $g$ on $M$ with the following properties:

(a) $g$, being restricted to the $\mu$-ellipsoids, coincides there with the locally defined euclidean metric $g_{E}$,

(b) everywhere in $M_{\omega}^{\circ}$ the distributions $K_{\omega}$ and $K_{\Omega}$ are orthogonal,

(c) $\omega_{\Omega}=h \cdot \mathcal{V}_{\Omega}^{1}$,

(d) $\Omega_{\omega}=h \cdot \mathcal{V}_{\omega}^{n-1}$,

(e) if $K_{\Omega}$ is tangent to the boundary $\partial M$, then $K_{\omega}$ is orthogonal to $\partial M$.

Proof. Since $\omega \wedge \Omega>0$ in $M_{\omega}^{\circ}, K_{\omega}$ and $K_{\Omega}$ are transversal. Furthermore, $\omega_{\Omega}=$ $\left.\omega\right|_{K_{\Omega}}$ and $\omega_{\Omega}=\left.\omega\right|_{K_{\Omega}}$ pick up the orientations of the corresponding subbundles. The same is true for the forms $\mathcal{V}_{\Omega}^{1}=h^{-1} \cdot \omega_{\Omega}$ and $\mathcal{V}_{\omega}^{n-1}=h^{-1} \cdot \Omega_{\omega}$. These orientations, taken together, agree with the preferred orientation of $T M$.

It is a crucial observation that in $B_{\mu, j}$ the volume forms $\mathcal{V}^{1}\left(\left.g_{E, j}\right|_{K_{\Omega}}\right)$ and $\mathcal{V}^{n-1}\left(\left.g_{E, j}\right|_{K_{\omega}}\right)$, induced by the euclidean metric, coincide respectively, with the forms $h^{-1} \cdot \omega_{\Omega}$ and $h^{-1} \cdot \Omega_{\omega}$. A similar conclusion is valid for the metrics $g_{E, k}$ in the half-ellipsoids $B_{k, \mu}^{+}$.

In constructing an appropriate $g$, we use the following simple extension principle. Given an oriented $k$-bundle $E \rightarrow N$ over a manifold $N$ with boundary $\partial N$, a $k$-form $\Theta>0$ in the fibers of $E$ and a Riemannian metric $g^{\partial}$ in the bundle $E \mid \rightarrow \partial N$ whose volume form $\mathcal{V}\left(g^{\partial}\right)$ coincides with the $\Theta$ over $\partial N$, there exists a metric $g$ in $E$ which extends $g^{\partial}$ and whose volume form $\mathcal{V}(g)=\Theta$ everywhere.

Indeed, one can extend $g^{\partial}$ to a metric $\tilde{g}$ on $E \rightarrow N$. The corresponding volume form $\mathcal{V}(\tilde{g})$ is proportional to $\Theta$ with a functional coefficient of proportionally $\gamma$ : $N \rightarrow \mathbf{R}_{+}$. Evidently, metric $g=\gamma^{1 / k} \cdot \tilde{g}$ does the job.

Hence, there exists a metric $g_{\omega}$ in the bundle $K_{\omega}$ over $M_{\omega, \mu}^{\circ}$ with the volume $(n-1)$-form $\mathcal{V}\left(g_{\omega}\right)=h^{-1} \cdot \Omega_{\omega}$. Similarly, there exists a metric $g_{\Omega}$ in the bundle $K_{\Omega}$ over $M_{\omega, \mu}^{\circ}$ with the volume 1 -form $\mathcal{V}\left(g_{\Omega}\right)=h^{-1} \cdot \omega_{\Omega}$. Moreover, by the extension principle, one can assume that, along the boundaries of the $\mu$-ellipsoids, $\mathcal{V}\left(g_{\omega}\right)=\mathcal{V}^{n-1}\left(\left.g_{E, j}\right|_{K_{\omega}}\right)$ and $\mathcal{V}\left(g_{\Omega}\right)=\mathcal{V}^{1}\left(\left.g_{E, j}\right|_{K_{\Omega}}\right)$. A similar property holds along the the boundaries of the half-ellipsoids.

Define $\tilde{g}$ on $M_{\omega, \mu}^{\star}$ to be the orthogonal sum of $g_{\omega}$ and $g_{\Omega}$. Notice that $K_{\omega}$ and $K_{\Omega}$ are orthogonal in the euclidean metrics around $S_{\omega}^{\star}$. Since $g_{\omega} \oplus g_{\Omega}$ coincides with the euclidean metrics over the boundaries of the $\mu$-ellipsoids, one can extend it across the ellipsoids to a metric $g$, well-defined on $M$.

Let $n$ be a vector field $g$-orthonormal to the boundary $\partial M$. The star operator $*_{g}^{\partial}$ in $\partial M$ is the contraction with $n$ of the Star operator $*_{g}$ in $M$, being restricted to $\partial M$. Therefore, $\left.\left.\Omega^{\partial}=*_{g}^{\partial}(\omega)=\{n\rfloor *_{g}(\omega)\right\}\left.\right|_{\partial M}=\{n\rfloor \Omega\right\}\left.\right|_{\partial M}$.

If $K_{\Omega}$ is tangent to $\partial M$, then $K_{\omega}$ is $g$-orthogonal to the boundary $\partial M$ and $n$ must be in $K_{\omega}$. Recall that, when the form $\left.\omega\right|_{\partial M}$ is non-singular, then we are able to construct $\Omega$ with its kernel tangent to the boundary. In this case, we have 
orthogonal decompositions: 1) $K_{\omega}=K_{\omega}^{\partial} \oplus\{n\}$, where $K_{\omega}^{\partial}=K_{\omega} \cap T(\partial M)$; 2) along the boundary, $K_{\Omega^{\partial}}=K_{\Omega}$; and 3) $T(\partial M)=K_{\omega}^{\partial} \oplus K_{\Omega^{\partial}}$.

By the very construction of $\Omega$ and $n \in K_{\omega}$, the form $\left.\Omega^{\partial}=*_{g}^{\partial}(\omega)=\{n\rfloor \Omega\right\}\left.\right|_{\partial M}$ is closed. This fact, together with the orthogonal decompositions above, establishes the complete similarity between the settings in $M$ and in its boundary. Therefore, if $\left.\omega\right|_{\partial M}$ is non-singular, any argument about $\omega$ and $\Omega$ carried before or after, will apply to $\left.\omega\right|_{\partial M}$ and $\Omega^{\partial}$ in $\partial M$.

Now we would like to clarify the meaning of the auxiliary function $h-$ an important ingredient in the construction of $g$.

Using the splitting $K_{\omega} \oplus K_{\Omega}$ of the tangent bundle, the $(n-1)$-form $\mathcal{V}\left(g_{\omega}\right)$ extends from the subbundle $K_{\omega}$ over $M_{\omega}^{\circ}$ to the form $\tilde{\mathcal{V}}\left(g_{\omega}\right)=h^{-1} \cdot \Omega_{\omega}$, defined on $T M_{\omega}^{\circ}$. Analogously, the 1-form $\mathcal{V}\left(g_{\Omega}\right)$ extends from the subbundle $K_{\Omega}$ over $M_{\omega}^{\circ}$ to the form $\tilde{\mathcal{V}}\left(g_{\Omega}\right)=h^{-1} \cdot \omega_{\Omega}$ defined on $T M_{\omega}^{\circ}$. Notice that $K_{\tilde{V}\left(g_{\omega}\right)}=K_{\omega}$ and $K_{\tilde{V}\left(g_{\Omega}\right)}=K_{\Omega}$, which uniquely characterize the extensions.

By the definition of the Hodge star-operator $*_{g}$ and the orthogonality of $K_{\omega}$ and $K_{\Omega}$, we have $*_{g}\left[\tilde{\mathcal{V}}\left(g_{\omega}\right)\right]=\tilde{\mathcal{V}}\left(g_{\Omega}\right)$. Therefore, in $M_{\omega}^{\circ}$,

$$
*_{g}(\omega)=*_{g}\left(h \cdot \tilde{\mathcal{V}}\left(g_{\Omega}\right)\right)=h \cdot *_{g}\left(\tilde{\mathcal{V}}\left(g_{\Omega}\right)\right)=h \cdot \tilde{\mathcal{V}}\left(g_{\omega}\right)=\Omega .
$$

On the other hand, $*_{g}(\omega)=\Omega$ in the $\mu$-ellipsoids. Consequently, $*_{g}(\omega)=\Omega$ everywhere in $M$.

Since by Lemma 2.2, (1), $\mathrm{d} \Omega=0$, this proves the harmonicity of $\omega$ and $\Omega$ in the metric $g=g_{\lambda \mu}$ and hence statement (2) from Theorem A.

The point-wise norm $\|\omega\|_{g}: M_{\omega}^{\circ} \rightarrow \mathbf{R}_{+}$satisfies the identity

$\left.\|\omega\|_{g}^{2} \cdot \mathcal{V}^{n}=\omega \wedge *_{g}(\omega)=\left(h \cdot \tilde{\mathcal{V}}\left(g_{\Omega}\right)\right) \wedge\left(h \cdot \tilde{\mathcal{V}}\left(g_{\omega}\right)\right)=h^{2} \cdot \tilde{\mathcal{V}}\left(g_{\Omega}\right)\right) \wedge\left(\tilde{\mathcal{V}}\left(g_{\omega}\right)=h^{2} \cdot \mathcal{V}^{n}\right.$.

Hence, $h=\|\omega\|_{g}=\|\Omega\|_{g}$ everywhere in $M$. This retroactively motivates properties (1)-(4) in the definition (2.7) of $h$.

Let $X_{\omega}$ denote the gradient vector field on $M, g$-dual to $\omega$. It is characterized by the two properties:

1) In $M_{\omega}^{\circ}, \quad X_{\omega} \in K_{\Omega}$.

2) In $M, \quad \omega\left(X_{\omega}\right)=h^{2}$.

Note that $X_{\omega}$ along $\partial M_{\omega, 2 \epsilon}^{\circ}$ is tangent to the boundary. For a positive singularity $x \in S_{\omega,+}^{\partial}$ (i.e. $a_{1}$ being positive), along $B_{2 \epsilon, k}^{\partial}$ the vector field is directed inwards $M$ and for a negative singularity - outward. If $\left.\omega\right|_{\partial M}$ is non-singular, then $X_{\omega}$ is tangent to the boundary everywhere.

The harmonicity of $\omega$ implies that the divergence of $X_{\omega}$ is zero:

$$
\left.\left.\operatorname{div} X_{\omega}=\mathrm{d}\left\{X_{\omega}\right\rfloor \mathcal{V}^{n}(g)\right\}=\mathrm{d}\left\{X_{\omega}\right\rfloor\left(h^{-2} \cdot \omega \wedge \Omega\right)\right\}
$$


and, since $\left.X_{\omega}\right\rfloor \Omega=0$,

$$
\left.\operatorname{div} X_{\omega}=\mathrm{d}\left\{h^{-2} \cdot\left(X_{\omega}\right\rfloor \omega\right) \cdot \Omega\right\}=\mathrm{d} \Omega=0 .
$$

Hence, the flow generated by $X_{\omega}$ is the $g$-volume-preserving.

Let $\mathcal{L}_{\omega}$ denote the Lie derivative along the field $X_{\omega}$. Then

$$
\left.\left.\mathcal{L}_{\omega}(\omega)=X_{\omega}\right\rfloor \mathrm{d} \omega+\mathrm{d}\left(X_{\omega}\right\rfloor \omega\right)=\mathrm{d}\left(h^{2}\right) .
$$

Therefore, outside of the $2 \mu$-ellipsoids, $\omega$ is invariant under the $X_{\omega}$-flow. In fact, $h^{2}$ can serve as a Lyapunov function for the $X_{\omega}$-flow. Note that inside of the ellipsoids $\omega$ is not $X_{\omega}$-invariant. On the other hand, since $\mathrm{d} \Omega=0$ and $X_{\omega} \in K_{\Omega}$,

$$
\left.\left.\mathcal{L}_{\omega}(\Omega)=X_{\omega}\right\rfloor \mathrm{d} \Omega+\mathrm{d}\left(X_{\omega}\right\rfloor \Omega\right)=0,
$$

which implies the $X_{\omega}$-invariance of $\Omega$. In other words, $\Omega$ can serve as a smooth invariant transversal measure for the singular 1-foliation $\mathcal{F}_{\Omega}$. This proves claim (2) from Theorem $\mathrm{A}^{\perp}$.

Now we are going to establish the near-minimality of the singular foliations $\mathcal{F}_{\omega}, \mathcal{F}_{\Omega}$ and to derive a few implications of this fact.

Let $F$ be a finite union of compact non-singular $\mathcal{F}_{\omega}$-leaves which do not intersect the $2 \mu$-ellipsoids around $S_{\omega}^{\star}$. In general, $F$ is a compact manifold with a boundary $\partial F \subset \partial M_{\omega, 2 \mu}^{\circ}$.

We shall compare its $g=g_{\lambda, \mu}$-induced $(n-1)$-volume $V(F)$ with the volume of any other $(n-1)$-dimensional pseudo-manifold $(\Sigma, \partial \Sigma) \hookrightarrow(M, \partial M)$, subject to condition

$$
\int_{\Sigma} \Omega=\int_{F} \Omega
$$

We are interested in situations where condition (2.8) is of a homological nature. Since $\mathrm{d} \Omega=0$, in particular, this happens when

$$
\partial \Sigma=\partial F \quad \text { and } \Sigma, F \text { are homologous modulo } \partial F, \text { or when }
$$

$S_{\omega}^{\partial}=\phi\left(\right.$ which implies $\left.\left.\Omega\right|_{\partial M}=0\right)$ and $\Sigma, F$ are homologous modulo $\partial M$.

In any case, we notice that the form

$$
\tilde{\Omega}=\Omega-\sum_{k} \Omega_{k}^{+}=\sum_{j} \Omega_{j}+q \cdot \Omega^{\dagger}
$$

(see (2.4)), as well as the form $\tilde{\Omega}^{\dagger}=q \cdot \Omega^{\dagger}$, are cohomologous to $\Omega$, thus realizing the same class $[\Omega] \in H^{n-1}(M ; \mathbf{R})$ (recall that the forms $\Omega_{j}$ 's and $\Omega_{k}^{+ \text {'s }}$ are exact). At the same time, $\left.\tilde{\Omega}\right|_{\partial M}=0$. Therefore, $\tilde{\Omega}$ gives rise to an element 
$[\tilde{\Omega}] \in H^{n-1}(M ; \partial M ; \mathbf{R})$. Note that the difference between $\Omega$ and $\tilde{\Omega}$ diminishes as $\epsilon \rightarrow 0$. Furthermore, not only, the difference $\Omega-\tilde{\Omega}$ is exact, but there exists an $(n-2)$-form $\Psi$, such that $\Omega-\tilde{\Omega}=\mathrm{d} \Psi$ and $\Psi$, being restricted to $\partial M$, is supported in the disjoint union of the ellipsoids $B_{2 \epsilon, k}^{\partial}$ 's. Therefore, for a relative cycle $\Sigma$,

$$
\int_{\Sigma} \Omega=\int_{\Sigma} \tilde{\Omega}+\int_{\partial \Sigma} \Psi
$$

Hence, if $\partial \Sigma \cap\left(\sqcup_{k} B_{2 \epsilon, k}^{\partial}\right)=\phi$, then $\int_{\Sigma} \Omega=\int_{\Sigma} \tilde{\Omega}$. Since one can isotope $\Sigma$ so that $\partial \Sigma$ will miss the ellipsoids $B_{2 \epsilon, k}^{\partial}$ 's, any $\Sigma$ can be isotoped to a $\Sigma^{\prime}$ for which $\int_{\Sigma^{\prime}} \Omega=\int_{\Sigma^{\prime}} \tilde{\Omega}$. Using that $\left.\tilde{\Omega}\right|_{\partial M}=0$, the correspondence $\Sigma \Rightarrow \int_{\Sigma} \Omega$ is a welldefined functional on $H_{n-1}(M ; \partial M ; \mathbf{Z})$ and thus, on $H_{n-1}(M ; \partial M ; \mathbf{R})$. Therefore, $\int_{\Sigma} \Omega=\int_{F} \Omega$ is a well-defined homological condition imposed on a relative cycle $\Sigma$, despite the fact that $\left.\Omega\right|_{\partial M}$ is not identically zero.

Recall that $F$ is comprised of $\mathcal{F}_{\omega}$-leaves; hence,

$$
V(F)=\int_{F} \tilde{\mathcal{V}}\left(g_{\omega}\right)=\int_{F} h^{-1} \cdot \Omega .
$$

Since $h=\lambda$ identically in $M_{\omega, 2 \mu}^{\star}$ and $F \subset M_{\omega, 2 \mu}^{\star}$, we get $V(F)=\lambda^{-1} \int_{F} \Omega$. By $(2.8)$,

$$
V(F)=\lambda^{-1} \int_{\Sigma} \Omega
$$

We divide $\Sigma$ into two parts: $\Sigma_{\mu}^{\star}=\Sigma \cap M_{\omega, 2 \mu}^{\star}$, belonging to the exterior of the $2 \mu$ ellipsoids, and its complement $\Sigma_{\mu}^{S}$, lying in their interiors. In the new notations,

$$
V(F)=\lambda^{-1} \int_{\Sigma_{\mu}^{\star}} \Omega+\lambda^{-1} \int_{\Sigma_{\mu}^{S}} \Omega .
$$

We shall estimate separately the two terms in the right-hand side of (2.10). First,

$$
\lambda^{-1} \int_{\Sigma_{\mu}^{\star}} \Omega=\int_{\Sigma_{\mu}^{\star}} h^{-1} \Omega=\int_{\Sigma_{\mu}^{\star}} \tilde{\mathcal{V}}\left(g_{\omega}\right) .
$$

If the hyperplane $T_{x} \Sigma$, tangent to $\Sigma$ at $x$, is distinct from $K_{\omega, x}$, then the $g$-induced volume of an infinitesimal $(n-1)$-parallelepiped in $T_{x} \Sigma$ is strictly smaller than the volume of its orthogonal projection on $K_{\omega, x}$. The volume of the projection is captured by evaluating $\tilde{\mathcal{V}}\left(g_{\omega}\right)$ on the parallelepiped. Therefore, $\int_{\Sigma_{\mu}^{\star}} \tilde{\mathcal{V}}\left(g_{\omega}\right) \leq$ $V\left(\Sigma_{\mu}^{\star}\right)$, implying

$$
\lambda^{-1} \int_{\Sigma_{\mu}^{\star}} \Omega \leq V\left(\Sigma_{\mu}^{\star}\right)
$$


Now we estimate the contribution of the second term in (2.8):

$$
\left|\lambda^{-1} \int_{\Sigma_{\mu}^{S}} \Omega\right| \leq \lambda^{-1} \int_{\Sigma_{\mu}^{S}}\|\Omega\|_{\Sigma} d m_{\Sigma}
$$

where $d m_{\Sigma}$ denotes the $g$-induced measure on $\Sigma$ and $\|\Omega\|_{\Sigma}-$ the point-wise norm of $\Omega$, restricted to $\Sigma$, in the metric $\left.g\right|_{\Sigma}$. Recall that $\|\Omega\|=\|\omega\|=h \leq \lambda$. Since $\|\Omega\|_{\Sigma} \leq\|\Omega\|$, we get:

$$
\left|\lambda^{-1} \int_{\Sigma_{\mu}^{S}} \Omega\right| \leq \int_{\Sigma_{\mu}^{S}} d m_{\Sigma}=V\left(\Sigma_{\mu}^{S}\right)
$$

Therefore,

$$
V(F) \leq V\left(\Sigma_{\mu}^{\star}\right)+V\left(\Sigma_{\mu}^{S}\right)=V(\Sigma)
$$

This proves statement (1) from Theorem B.

The proof of statement (4) from Theorem A consists of practically the same argument: for a given compactly supported in $M_{\omega, 2 \mu}^{\star}$ perturbation $\mathcal{L}^{\prime}$ of an $\mathcal{F}_{\omega}$-leaf $\mathcal{L}$, one compares the volumes of compact domains $\mathcal{L}^{\prime} \backslash \mathcal{L}$ and $\mathcal{L} \backslash \mathcal{L}^{\prime}$ which share the same boundary and are cobordant modulo this boundary.

In order to prove (2) and (3) from Theorem B, we need to make a more careful comparison of the volumes of the cycle $F$ (built of leaf components) and a generic cycle $\Sigma$, subject to the homological constraint $\int_{\Sigma} \Omega=\int_{F} \Omega$ :

$$
V(F)=\int_{\Sigma_{\mu}^{\star}} \lambda^{-1} \Omega+\int_{\Sigma_{\mu}^{S}} \lambda^{-1} \Omega=\int_{\Sigma_{\mu}^{\star}} \cos \theta d m_{\Sigma}+\int_{\Sigma_{\mu}^{S}} \lambda^{-1} \Omega,
$$

where $\theta$ denotes the angle in the metric $g$ between the oriented line $K_{\Omega}$ and the normal to $\Sigma$. Now, if $V(\Sigma)=V(F)$, from the formula above we get:

$$
V\left(\Sigma_{\mu}^{\star}\right)-\int_{\Sigma_{\mu}^{\star}} \cos \theta \mathrm{d} m_{\Sigma}=\int_{\Sigma_{\mu}^{S}} \lambda^{-1} \Omega-V\left(\Sigma_{\mu}^{S}\right)
$$

Since $h \leq \lambda$, it follows that $\left\|\lambda^{-1} \Omega\right\|_{\Sigma} \leq\left\|\lambda^{-1} \Omega\right\|=\lambda^{-1} h \leq 1$. Therefore, the righthand side of (2.12) is non-positive. On the other hand, the left-hand side clearly is non-negative. Furthermore, if somewhere in $\Sigma_{\mu}^{\star}$, a portion of $\Sigma$ is transversal to the foliation $\mathcal{F}_{\omega}$, the left-hand side of (2.12) must be positive. Hence (2.12) implies that such a minimal $\Sigma$ has to "follow" the leaves in $\Sigma_{\mu}^{\star}$ and can diverge from them only in the $2 \mu$-ellipsoids. (In particular, if a connected $\Sigma$ as above has a non-empty intersection with a leaf $\mathcal{L}$, which misses the closed $2 \mu$-ellipsoids around $S_{\omega}^{\star}$, then $\Sigma=\mathcal{L}$ and $\mathcal{L}$ must be closed in $M$.) 
It also follows from the vanishing of the two sides of (2.12) that, for a volumeminimizing $\Sigma$, we must have $V\left(\Sigma_{\mu}^{S}\right)=\lambda^{-1} \int_{\Sigma_{\mu}^{S}} \Omega$ and $V\left(\Sigma_{\mu}^{*}\right)=\lambda^{-1} \int_{\Sigma_{\mu}^{\star}} \Omega$. This proves claim (2) from Theorem B.

With $F$ as in Theorem B being fixed, our next task is to estimate the variation of the volume for any finite union $\tilde{F}$ of compact leaf components, such that $\int_{\tilde{F}} \Omega=$ $\int_{F} \Omega$. Such an $\tilde{F}$ can penetrate inside the $2 \mu$-ellipsoids, where it could fail to be a volume-minimizing hypersurface. Therefore, we shall concentrate on the behavior of the volume $V\left(\tilde{F}_{\mu}^{S}\right)=\int_{\tilde{F}_{\mu}^{S}} h^{-1} \Omega$ as function of $\mu$ and $\lambda$.

Recall that, among a variety of choices, the construction of $\Omega$ depended on a choice of a parameter $\epsilon>0$. For $\mu<\delta(\epsilon) / 2$, in the ellipsoid $B_{\mu, j}, \Omega=*_{g_{E, j}}(\omega)$, where $\omega$ is described by (1.1). Similarly, in $B_{\mu, k}^{+}, \Omega=*_{g_{E, k}}(\omega)$, where $\omega$ is given by (1.3). Everywhere in $\left\{B_{2 \mu, j}\right\}$ and $\left\{B_{2 \mu, k}^{+}\right\}$the metric $g_{\lambda, \mu}$ is conformly equivalent to the euclidean metric $g_{E}$. In $\left\{B_{\mu, j}\right\}$ and $\left\{B_{\mu, k}^{+}\right\}$, the two metrics coincide, while in the shells $\left\{B_{2 \mu, j} \backslash B_{\mu, j}\right\},\left\{B_{2 \mu, k}^{+} \backslash B_{\mu, k}^{+}\right\}$the conformal factor $g_{\lambda, \mu} / g_{E}=h^{-1}\|\omega\|_{E} \leq 1$, provided $\lambda \geq 2 \mu$ (cf. (2.7) and Figure 6).

Therefore, when $\lambda \geq 2 \mu$,

$$
V\left(\tilde{F}_{\mu}^{S}\right)=\int_{\tilde{F}_{\mu}^{S}} h^{-1} \Omega \leq V_{E}\left(\tilde{F}_{\mu}^{S}\right)
$$

where $V_{E}(\sim)$ stands for the euclidean volume.

For a given $F$, there exists a universal and $\lambda \mu$-independent constant $\kappa_{F}$, such that, for any $\tilde{F}$ homologous to $F$, the intersection of $\tilde{F}$ with each of the $2 \mu$-ellipsoids consists not more than of $\kappa_{F}$ components of the foliation. To see it, let us install a measuring device at each of the singularities. The device is an $\operatorname{arc} \gamma_{j}^{\star}$ (or two), transversal to the foliation in the ellipsoid (if the Morse index of the singularity is 1 or $n-1$, then one needs two $\operatorname{arcs})$. The $\operatorname{arc}(\mathrm{s})$ starts and terminates at the boundary of the ellipsoid and pierces every leaf component lying in the ellipsoid. For example, one can take an arc entering the singularity along the stable disk and exiting it through the unstable one. By the Calabi property, one can complete $\gamma_{j}^{\star}$ to an $\omega$-positive loop $\gamma_{j}$. This loop is the measuring device we need. On one hand, the intersection number of $\tilde{F}$ with $\gamma_{j}$ is positive and exceeds the number of components of the $\tilde{F}$ in the ellipsoid. On the other hand, since $\tilde{F}$ and $F$ are homologous, $\tilde{F} \circ \gamma_{j}=F \circ \gamma_{j}$. Hence, pick $\kappa_{F}=\max _{j}\left\{F \circ \gamma_{j}\right\}$.

With this choice of $\kappa_{F}, V_{E}\left(\tilde{F}_{\mu}^{S}\right) \leq \tilde{K} \cdot \mu^{n-1}$, where $\tilde{K}>\kappa_{F} \cdot A$ and $A$ denotes the maximal euclidean volume of $\mathcal{F}_{\omega}$-leaf components in the $\mu$-ellipsoid, divided by $\mu^{n-1}$.

Now, since

$$
V(F)=\int_{F} \lambda^{-1} \Omega=\int_{\tilde{F}} \lambda^{-1} \Omega=\int_{\tilde{F}_{\mu}^{\star}} \lambda^{-1} \Omega+\int_{\tilde{F}_{\mu}^{S}} \lambda^{-1} \Omega
$$


and

$$
V(\tilde{F})=\int_{\tilde{F}} h^{-1} \Omega=\int_{\tilde{F}_{\mu}^{\star}} \lambda^{-1} \Omega+\int_{\tilde{F}_{\mu}^{S}} h^{-1} \Omega,
$$

for a constant $\tilde{K}$ (depending on $F$, not on $\tilde{F}$ ), we get:

$$
|V(\tilde{F})-V(F)|=\left|\int_{\tilde{F}_{\mu}^{S}}\left(h^{-1}-\lambda^{-1}\right) \Omega\right| \leq\left|\int_{\tilde{F}_{\mu}^{S}} h^{-1} \Omega\right|=V\left(\tilde{F}_{\mu}^{S}\right) \leq \tilde{K} \cdot \mu^{n-1} .
$$

This proves claim (3) of Theorem B and, finally, completes the proof of the Theorem B as a whole.

At the same time, these calculations and estimates imply statements (1)-(3) of Proposition 1.6. Indeed, by the very construction of the metrics $g_{\lambda, \mu}, V\left(M_{\omega, 2 \mu}^{\star}\right)=$ $\lambda^{-2} \int_{M_{\omega, 2 \mu}^{\star}} \omega \wedge \Omega$ and, since

$$
V\left(M \backslash M_{\omega, 2 \mu}^{\star}\right) \geq V_{E}\left(\coprod_{j} B_{\mu, j} \cup \coprod_{k} B_{\mu, k}^{+}\right)=c \cdot \mu^{n}
$$

(c being a universal constant), as $\lambda \rightarrow \infty$, the volume of the $2 \mu$-ellipsoids approaches the volume $V(M)$ as $\sim \lambda^{-2}$. Moreover, as $\lambda$ grows, the Riemannian metric $g_{\lambda, \mu}$ uniformly declines in $M_{\omega, 2 \mu}^{\star}$ and stabilizes in the $\mu$-ellipsoids. Therefore, when $\lambda \rightarrow \infty$, the spaces $\left\{M, g_{\lambda, \mu}\right\}$ converge, in the Gromov's sense, to a compact metric space which topologically is a bouquet of a few spheres and hemispheres. The spheres are indexed by the singularities of $\omega$ and the hemispheres by the singularities of $\left.\omega\right|_{\partial M}$. The limit metric on the spheres depends only on the Morse types of the singularities and on the choice of the auxiliary function $h$.

On the other hand, for a fixed $\lambda$, as $\mu \rightarrow 0$, the volume of the $2 \mu$-ellipsoids declines at the rate $\sim \mu^{n}$ - the coefficients of $h^{-2} \cdot \omega \wedge \Omega$ are bounded in the ellipsoids. At the same time, $V(F)=\lambda^{-1} \int_{F} \Omega$. Therefore,

$$
\lim _{\mu \rightarrow 0} V(M)=\lambda^{-2} \int_{M} \omega \wedge \Omega
$$

and, for any $\tilde{F}$ as above,

$$
\lim _{\mu \rightarrow 0} V(\tilde{F})=\lambda^{-1} \int_{F} \Omega
$$

This ends the proof of Proposition 1.6.

Now we are in the position to prove Theorems $\mathrm{A}^{\perp}, \mathrm{B}^{\perp}$ and Corollary 1.5. The arguments are very similar to the ones used in proving Theorems A, B and Proposition 1.6. The only crucial difference is that, prior to dealing with the 1foliation $\mathcal{F}_{\Omega}$, we can be more flexible with the choice of $\Omega-$ unlike $\omega$, it is not a 
given, but rather handmade contraption. In particular, if $n>2$, given any finite collection of $\omega$-positive loops $\left\{\gamma_{r}\right\}$, by Lemma 2.1, it is possible to construct an $\omega$-subordinate cover $\mathcal{U}=\left\{U_{l}\right\}$, so that each loop $\gamma_{r}$ is surrounded by an $\omega$-positive tube $V_{r} \subset U_{r}$ which does not intersect the rest of the elements from $\mathcal{U}$. Moreover, the trivialization of $V_{r}$ can be chosen to agree with the foliation $\mathcal{F}_{\omega}$ - the $(n-1)$ disks, normal to $\gamma_{r}$, are contained in the leaves of $\mathcal{F}_{\omega}$. In such a trivialization of $V_{r}$, the $\mathcal{F}_{\Omega^{-}}$-leaves are the longitudes of the solid torus $h_{r}: S^{1} \times D^{n-1} \approx V_{r}$, the form $\omega=\left(h_{r}^{-1}\right)^{*}(d t)$ and $\Omega=\left(h_{r}^{-1}\right)^{*}\left(d u_{1} \wedge \cdots \wedge d u_{n-1}\right)$, where $\left\{t, u_{1}, \ldots, u_{n-1}\right\}$ are the standard coordinates in $S^{1} \times D^{n-1}$. With this choice of $\omega$ and $\Omega$, the corresponding metric $g=g_{\omega} \oplus g_{\Omega}$ is the standard flat metric in the flat solid torus $S^{1} \times D^{n-1}$ and all the longitudes are geodesics.

Consider a leaf $L$ of $\mathcal{F}_{\Omega}$ and its finite $\operatorname{arc} L^{\prime}$. The arc length of $L^{\prime}$ is given by integrating $h^{-1} \omega$ along the arc. If the $\operatorname{arc} L^{\prime}$ is missing the $2 \mu$-ellipsoids, its length $l\left(L^{\prime}\right)=\lambda^{-1} \int_{L^{\prime}} \omega$. Let $\gamma$ be any arc close to $L^{\prime}$ and having the same ends. Since $\omega$ is closed, $\lambda^{-1} \int_{L^{\prime}} \omega=\lambda^{-1} \int_{\gamma} \omega$. By an argument, similar to the one we have used to prove the intrinsic minimality of the foliation $\left.\mathcal{F}_{\omega}\right|_{M_{\omega, 2 \mu}^{\circ}}$, the latter integral does not exceed the length $l(\gamma)$. Thus, $L^{\prime}$ is a geodesic arc. In a similar way, one can prove that, if $L$ is a closed leaf (a loop) in $M_{\omega, 2 \mu}^{\circ}$ and $\gamma$-any loop such that $\int_{\gamma} \omega=\int_{L} \omega$, then $l(\gamma) \geq l(L)$. The proof of statement (4) of Theorem $\mathrm{A}^{\perp}$ is very similar to the one, connecting formula (2.10) with the conclusion of the proof of Theorem A.

The arguments that prove (2) and (3) from Theorem $\mathrm{B}^{\perp}$ are an 1-dimensional version of the arguments that led to formula (2.12) and its implications. This completes the proof of Theorems $\mathrm{A}^{\perp}, \mathrm{B}^{\perp}$.

The considerations above demonstrate that, given a finite collection of $\omega$ positive loops $\left\{\gamma_{r}\right\}$, there exists metric $g_{\lambda, \mu}$ with the properties described in Theorems $\mathrm{A}^{\perp}, \mathrm{B}^{\perp}$ and such that the $\gamma_{r}$ 's are geodesics and closed leaves of $\mathcal{F}_{\Omega}$. Jointly, (with $\lambda=1$ ), the two arguments prove Corollary 1.5.

Now we are in position to sketch the proof of Proposition 2.5. The idea is to build large bumps of metric, supported in the $\omega$-positive tubes $U_{l}$ surrounding the given loops $\gamma_{l}$. The bumps will be constructed along the directions transversal to the loops to make it very costly for a minimal hypersurface to cut through the loops. This will minimize the average number of cuts among the geodesic loops comprising $U_{l}$ to the absolute homological necessity.

By Corollary 1.5, we can assume the existence of a metric $g_{0}$ in which $\omega$ is harmonic and the non-intersecting tubes $U_{l}$ being comprised of $\omega$-positive geodesic loops $\gamma_{l}^{\prime}$ of the fixed length $\int_{\gamma_{l}} \omega$.

To simplify the notations, we drop the index $l$ enumerating the tubes and concentrate on a particular tube $U$ with the core $\gamma$.

Denote by $\Omega_{U}$ a closed $(n-1)$-form supported in $U$ and whose kernels are tangent to the $\omega$-positive longitudes of the tube. By adding the forms $q \cdot \Omega_{U}$ to the previously built form $\Omega$ - the central ingredient in constructing $g_{0}-$, we will 
create a $q$-parameterized family of closed $(n-1)$-forms $\Omega_{q}=\Omega+q \Omega_{U}$ and the corresponding family of metrics $g_{q}$. For large $q$, all the normal to $\gamma$ disks $D$ (the fibers of the tube $U$ over $\gamma$ ) will be uniformly large in $g_{q}$. At the same time, in the metric $g_{q}$, the longitudes of the solid torus $U \approx D \times S^{1}$ remain to be geodesics and their lengths do not change.

Let an oriented hypersurface $\Sigma_{q}$ be the volume-minimizing cycle in its relative homology class $[\Sigma]$. By $[\mathrm{Fe}]$, such a cycle can be realized by a pseudo-manifold with the singular set at least of codimension 7. (Some of the components of such minimal hypersurface $\Sigma_{q}$ might occur with multiplicity greater than one.)

Clearly, each geodesic loop $\gamma^{\prime}$ from $U$ which hits $\Sigma_{q}$ transversally, must hit it at least $d=[\Sigma] \circ \gamma$ times, however, some loops might have more than $|d|$ transversal intersections with $\Sigma_{q}$. The algebraic intersection numbers can be interpreted as local degrees of an obvious map $\pi$ from the portion $\Sigma_{q} \cap U$ into the $(n-1)$-disk $D$ - a typical fiber of $U$ over $\gamma$. Let us exclude the $\pi$-critical value set from $D$ (this set of measure zero contains the $\pi$-image of the singular set of $\Sigma_{q}$ ). The rest of $D$ can be divided in two complementary sets: $A$, formed by regular values with exactly $|d|$ preimages, and $B$, formed by regular values with more than $|d|$ preimages. Proving that $A$ is non-empty, will be a significant step in the proof of the proposition. In fact, using the minimality of $\Sigma_{q}$ we will show that, as $q \rightarrow \infty$, the ratio of the $g_{q}$-induced measure $m(B)$ of $B$ to the measure $m(A)$ of $A$ tends to zero.

Indeed, since $\pi$ is an orthogonal projection, the volume of $\Sigma_{q}^{U}:=\Sigma_{q} \cap U$ has to exceed $|d| \cdot m(A)+(|d|+1) \cdot m(B)$. We shall compare this volume with the volume of another cycle $\Delta_{q}$, homologous to $\Sigma_{q}$. It will be assembled of three parts, the first of which is the portion $\Sigma_{q}^{\circ}$ of $\Sigma_{q}$ outside of the tube $U$. The second part consists of (properly oriented) $|d|$ disjoint fibers $\left\{D_{i}\right\}$ of the disk bundle $U \rightarrow \gamma$. The third - of the (singular) cobordism $W_{q}$ in $\partial U$ between $\Sigma_{q} \cap \partial U$ and the union of $|d|$ spheres $S_{i}:=D_{i} \cap \partial U$.

The volume of $\Delta_{q}$ is the sum of the corresponding three volumes: $V\left(\Sigma_{q}^{\circ}\right)$, $V\left(W_{q}\right)$ and $|d| \cdot V(D)$. Since $V\left(\Sigma_{q}\right) \leq V\left(\Delta_{q}\right)$, one must have

$$
|d| \cdot m(A)+(|d|+1) \cdot m(B) \leq V\left(W_{q}\right)+|d| \cdot V(D) .
$$

Note that the volume $V\left(W_{q}\right)$ is bounded from above by the $|d|$-multiple of the volume of $\partial U$ and therefore is $q$-independent. Since $m(A)+m(B)=V(D)$, we can rewrite the inequality above as $m(B) \leq V\left(W_{q}\right)$, which provides a $q$-independent upper bound for $m(B)$.

Since $\lim _{q \rightarrow \infty} V(D)=\infty$, we conclude that $\lim _{q \rightarrow \infty} m(B) / V(D)$ must be zero. Therefore, as $q \rightarrow \infty$, the vast majority of the geodesic loops from $U$ will hit $A$ and will have the minimal possible number of intersections with the volume minimizing cycles in the homology class $[\Sigma]$. The same conclusion is valid for any finite set of homology classes $\left\{\left[\Sigma_{k}\right] \in H_{n-1}(M, \partial M ; \mathbf{Z})\right\}_{k}$.

In the argument leading to Proposition 2.4, we noticed that, for any nontrivial class $[\omega] \in H^{1}(M ; \mathbf{R})$, there exists a representative intrinsically harmonic 
form $\omega$, such that one can find a basis in $H_{1}(M ; \mathbf{Z}) /$ Tor represented by $\omega$-positive loops. Applying previous arguments to such loops and a generating set of elements $\left[\Sigma_{k}\right] \in H_{n-1}(M, \partial M ; \mathbf{Z})$, shows the existence of a metric in which both bases are realized by the volume-minimizing cycles (of dimensions 1 and $(n-1)$ ) and their mutual intersections have the minimal cardinalities consistent with the homology intersection pairings. This proves the proposition.

\section{Absolute case}

Definition 3.1. We say that a 1 -form $\omega$ on $M$ satisfies the absolute Calabi condition, if through each point $x \in M_{\omega}^{\circ}$, there exists an $\omega$-positive path which is closed or, alternatively, which starts and terminates at points of the boundary $\partial M$. Moreover, at the start and the end points the path is transversal to the boundary.

For example, the form $\omega$, created with the help of Figure 1b satisfies this definition: just take a look at the Calabi graph $\Gamma_{\omega}(M)$ in Figure 2b. For closed manifolds, the relative and absolute Calabi properties coincide; however, in general, the relative condition implies the absolute one.

The natural pairing $\star: H^{n-1}(M ; \mathbf{Z}) \otimes H_{n-1}(M ; \mathbf{R}) \rightarrow \mathbf{R}$ can be induced by integrating a closed $(n-1)$-form $\Omega$ over a closed pseudo-manifold $\Sigma \hookrightarrow M$.

Here are the absolute versions of Theorems A and B.

Theorem C. Let $M, \lambda, \mu$ be as in Theorem $A$ and let $\omega$ be a closed Morse-type 1-form satisfying the absolute Calabi condition. Then there exists a $\lambda \mu$-family of Riemannian metrics $g_{\lambda, \mu}$, so that the following claims hold:

(1) All the metrics $g_{\lambda, \mu}$ are conformally equivalent. For any $\mu^{\prime} \leq \mu$, the metrics $g_{\lambda, \mu}$ and $g_{\lambda, \mu^{\prime}}$ coincide on the complement to the $\mu$-ellipsoids, centered on the singularities of $\omega$.

(2) The form $\omega$ is harmonic with respect to $g_{\lambda, \mu}$.

(3) The closed $(n-1)$-form $\Omega=*_{g_{\lambda}, \mu}(\omega)$ is $\lambda, \mu$-independent. The form $\Omega$ gives rise to a non-trivial class $[\Omega] \in H^{n-1}(M ; \mathbf{R})$.

(4) All the leaves of the foliation $\mathcal{F}_{\omega}$, outside of the $\mu$-ellipsoids, are minimal hypersurfaces in the metric $g_{\lambda, \mu}$.

Theorem D. Under assumptions and notations of Theorem $C$, the following claims are valid:

(1) Let $F$ be any finite union of compact leaf components of the foliation $\mathcal{F}_{\omega}$, represented by closed manifolds not intersecting the $\mu$-ellipsoids around the singularities. Then $F$ minimizes the $g_{\lambda, \mu}$-induced $(n-1)$-volume $V(F)$ among all closed pseudo-manifolds $\Sigma \hookrightarrow M$, subject to the homological constraint $[\Omega] \star[\Sigma]=[\Omega] \star[F]$. 
In particular, $F$ minimizes the volume in its homology class. This minimal volume $V(F)=\lambda^{-1}[\Omega] \star[F]$.

(2) Any closed pseudo-manifold $\Sigma \hookrightarrow M$, of the volume $V(F)$ and such that $[\Omega] \star[\Sigma]=[\Omega] \star[F]$, outside of the $\mu$-ellipsoids, is comprised of compact leaves of the foliation $\mathcal{F}_{\omega}$, being restricted to the exterior of the ellipsoids. The volume of the portion $\Sigma_{\mu}^{S}$ of $\Sigma$, lying inside of the $\mu$-ellipsoids, is given by the integral $\lambda^{-1} \int_{\Sigma_{\mu}^{S}} \Omega$. When $\lambda \rightarrow+\infty$, it declines as $\sim \lambda^{-1}$; when $\mu \rightarrow 0$, it declines as $\sim \mu^{n-1}$.

(3) For any union $\tilde{F}$ of compact leaf components, represented by closed manifolds (possibly, with the Morse-type singularities) and homologous to $F$, the volume variation $|V(\tilde{F})-V(F)| \leq K \cdot \mu^{n-1}$, where $K$ is a positive, $\lambda \mu$-independent constant and $\lambda \geq \mu$.

Remark. Here the requirement on $F, \tilde{F}, \Sigma$ to be closed is essential. For example, take the form $\omega$ created in Figure 1b. Consider the leaf component of $\mathcal{F}_{\omega}$ represented by a short chord $F$ of the boundary curve, where $\partial M$ is shaped as letter $\mathcal{Z}$. Clearly, it defines a trivial relative cycle and is not a length-minimizing arc.

Theorems C and D, like Theorems A and B, have their "Poincaré-dual" analogs:

Theorem $\mathbf{C}^{\perp}$. Under the assumptions and notations of Theorem $C$, claims (1)(3) of Theorem $A^{\perp}$ are valid.

Theorem $\mathbf{D}^{\perp}$. Under the assumptions and notations of Theorem $C$, the following statements hold:

(1) Let $L$ be any finite collection of compact closed $\mathcal{F}_{\Omega}$-leaves that does not intersect the $\mu$-ellipsoids around the $\omega$-singularities. Then $L$ minimizes the $g_{\lambda, \mu^{-}}$ induced length $l(L)$ among all the 1-cycles $\Gamma$ in $M$, subject to the homological condition $\int_{\Gamma} \omega=\int_{L} \omega$. In particular, $L$ minimizes the length in its homology class. The minimal length $l(L)=\lambda^{-1} \int_{L} \omega$.

(2) Any 1-cycle $\Gamma$ of the length $l(L)$ and such that $\int_{\Gamma} \omega=\int_{L} \omega$, outside of the $\mu$-ellipsoids, is comprised of a number of compact leaf components of $\mathcal{F}_{\Omega}$, restricted to the exterior of the ellipsoids (outside of the ellipsoids, $\Gamma$ consists of geodesic arcs).

The length of the portion $\Gamma_{\mu}^{S}$ of $\Gamma$, lying inside of the $\mu$-ellipsoids, is given by the integral $\lambda^{-1} \int_{\Gamma_{\mu}^{S}} \omega$. When $\lambda \rightarrow+\infty$, it declines as $\sim \lambda^{-1}$; when $\mu \rightarrow 0$, it declines as $\sim \mu$.

(3) For any union $\tilde{L}$ of compact $\mathcal{F}_{\Omega}$-leaf components, represented by closed loops, and homologous to $L$, the length variation $|l(\tilde{L})-l(L)| \leq K \cdot \mu$, where $K$ is a positive, $\lambda \mu$-independent constant and $\lambda \geq \mu$.

(4) If $\left.\omega\right|_{\partial M}=0$, then the same conclusions apply to any relative 1 -cycles $L, \Gamma$, subject to $\int_{\Gamma} \omega=\int_{L} \omega$. 
Proof. Proofs of Theorems $\mathrm{C}, \mathrm{D}$ and $\mathrm{C}^{\perp}, \mathrm{D}^{\perp}$ are slight modifications of the arguments used in proving Theorems $\mathrm{A}, \mathrm{B}$ and $\mathrm{C}^{\perp}, \mathrm{D}^{\perp}$. The only difference is that, instead of using toroidal and half-toroidal $\omega$-positive tubes as in (2.1) and (2.2), one uses toroidal tubes together with cylindrical $\omega$-positive tubes $h_{l}:[0,1] \times D^{n-1} \rightarrow$ $U_{l} \subset M$ that start and terminate at points of $\partial M_{\omega}^{\circ}$. As a cylindrical tube transversally approaches the boundary $\partial M$, it is cut by $\partial M$ in a "slanted" fashion with respect to the $\mathcal{F}_{\omega}$-leaves as shown in Figure 7.

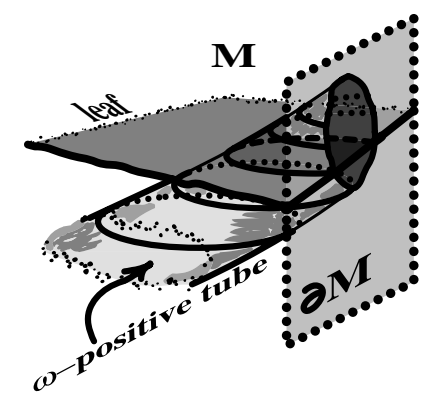

Figure 7

The singularities of $\left.\omega\right|_{\partial M}$ are treated as before: they contribute the halfellipsoids to the $\omega$-subordinate cover $\mathcal{U}$.

Corollary 3.1. Fix $\epsilon>0$. Let $f: M^{n} \rightarrow \mathbf{R}$ be a Morse function with no local maxima and minima inside $M$ and no critical points on the boundary $\partial M=$ $\partial M_{+} \amalg \partial M_{-}$. Assume that $f\left(\partial M_{+}\right)>f\left(\partial M_{-}\right)$. Let a be any $f$-regular value inbetween $f\left(\partial M_{+}\right)$and $f\left(\partial M_{-}\right)$. Let $F$ be a closed $(n-1)$-submanifold of $f^{-1}(a)$.

Then there exists a Riemannian metric $g=g_{\epsilon}$ on $M$, such that:

(1) $f$ is harmonic with respect to $g$.

(2) The $(n-1)$-form $\Omega=*_{g}(d f)$ is harmonic and represents a non-trivial element $[\Omega] \in H^{n-1}(M ; \mathbf{R})$.

(3) F minimizes the $g$-induced volume among all absolute $(n-1)$-cycles $\Sigma \hookrightarrow$ $M$, subject to the constraint $[\Omega] \star[\Sigma]=[\Omega] \star[F]$. Thus, $F$ is the volume-minimizing cycle in its homology class. In particular, if $F=f^{-1}(a)$, then it realizes the minimal volume among all constant level hypersurfaces $\tilde{F}=f^{-1}(b)$, where $b$ ranges in-between $f\left(\partial M_{+}\right)$and $f\left(\partial M_{-}\right)$. The volume variation among such $\tilde{F}$ 's is smaller than $\epsilon$.

(4) For any $c$, the hypersurface $f^{-1}(c)$ is minimal outside of the $\epsilon$-ellipsoids centered on the critical points of $f$ and the $\epsilon$-half-ellipsoids centered on the critical points of $f: \partial M \rightarrow \mathbf{R}$.

(5) The f-gradient flow preserves both the $g$-induced volume and the form $\Omega$. Its trajectories outside of the ellipsoids are geodesics. 
Proof. Condition " $f\left(\partial M_{+}\right)>f\left(\partial M_{-}\right)$", coupled with the fact that $f$ has no local maxima and minima inside $M$, implies the existence of an $d f$-positive path $\gamma_{x}$ through any given point $x \in M_{d f}^{\circ}$, such that $\gamma_{x}$ starts and terminates at a point in $\partial M$. Indeed, the ban on the critical points of indices 0 and $n$ make it possible to redirect $\gamma_{x}$, as it approaches a singularity $y$, from the set $f^{-1}(<f(y))$ to the ground higher than $f(y)$.

Now we can apply Theorems $\mathrm{C}, \mathrm{D}$ and $\mathrm{C}^{\perp}, \mathrm{D}^{\perp}$ to $\omega=d f$. For a given $F$, we choose $\mu$ to satisfy three conditions: 1) $2 \mu<\delta(\epsilon)<\epsilon$; 2) $K \cdot \mu^{n-1}<\epsilon$ (cf. Theorems C, D); 3) $F \subset M_{\omega, 2 \mu}^{\circ}$.

\section{Generalizations, questions and conjectures}

Let $C^{i, j}$ denote the cone in the euclidean space $\mathbf{R}^{i+1} \times \mathbf{R}^{j+1}$, given by $j\|x\|^{2}=$ $i\|y\|^{2}$, where $x \in \mathbf{R}^{i+1}, y \in \mathbf{R}^{j+1}$. Put $f(x, y)=j\|x\|^{2}-i\|y\|^{2}$, so that $f$ is a Morse function of index $j+1$ and $f^{-1}(0)=C^{i, j}$ is a linear cone over the product $S^{i} \times S^{j}$ of two spheres. It has been shown in $[\mathrm{Si}]$ that this cone is a minimal hypersurface in the euclidean metric $d g_{E}^{2}=\|x\|^{2}+\|y\|^{2}$, provided $i+j>5$ and $i \cdot j>5$. Furthermore, Hardt and Simon proved [HS] that any area minimizing hypercone in the euclidean space, smooth near infinity, gives rise to a family of minimal hypersurfaces forming a foliation with the cone as the only singular leaf.

However, the Morse function $f$ above is harmonic only when $i=j$. We do not know whether there are other pairs $(i, j)$, for which the Morse function $f$ : $\mathbf{R}^{i+j+2} \rightarrow \mathbf{R}$ of index $j+1$ at the origin is harmonic and defines a minimal foliation with respect to some Riemannian metric. If such a function exists, we shall call a pair $(n, j+1)=(i+j+2, j+1)$ harmonic. For example, by [Si], any pair $(2 k, k)$ is harmonic for $k \geq 4$.

Even when, for a Calabi form $\omega$, all the dimension-index pairs are harmonic, we do not know how to prove the analogs of Theorems A, B, C, D: our constructions seem to fail the minimality of the foliation $\mathcal{F}_{\omega}$ in the $2 \mu$-shells surrounding the $\omega$-singularities.

Conjecture 4.1. If all the dimension-index pairs of $\omega$-critical points are harmonic, then the results of this paper, dealing with the foliation $\mathcal{F}_{\omega}$, are valid "for $\mu=0 "$, in other words, all the leaves of the foliation $\mathcal{F}_{\omega}$, in the appropriate metric, become "true" minimal hypersurfaces (in contrast with the $\mu$-controlled failure of their minimality studied in the paper). In particular, under these assumptions, the Calabi property insures both the harmonicity of the form $\omega$ and the minimality of the $\mathcal{F}_{\omega}$-leaves.

We suspect that the pairs $(n, 1)$ and $(n, n-1)$ can not be harmonic. Recall (cf. Corollary 1.1) that forms with no critical points of indices $0,1, n-1, n$ are automatically Calabi. Thus, any form with harmonic indices is probably automatically 
intrinsically harmonic.

The Morse-type nature of the $\omega$-singularities does not seem to be important for the validity of our arguments.

Conjecture 4.2. All the results of this paper are valid for closed 1-forms $\omega$ of the Bott-Morse type, subject to the Calabi conditions described in Definition 0.2, or in (0.2), or in Definition 3.1.

Note that the Calabi positive loop properties prevent the singular $k$-manifolds of $\omega$ from carrying normal bundles of indices 0 or $n-k$.

Another line of questions that seem to require a detailed study is related to more general boundary conditions. Recall that, in the presence of the relative Calabi property (cf. Definition 0.2) and assuming that $\left.\omega\right|_{\partial M}$ is non-singular, we were able to synchronize the harmonicity of the forms and the near-minimality of the foliations in $M$ and in $\partial M$ (cf. Theorem A, (5) and Theorem B, (4)).

Question 4.3. Are the analogs of Theorems $A$ and $B$ valid for a generic $\left.\omega\right|_{\partial M}$ with the Morse-type singularities? In particular, is the property (0.2) sufficient to insure the harmonicity of $\omega$ and $\left.\omega\right|_{\partial M}$ with respect to some metric on $M$ ?

The question seems to be of a delicate nature. It is possible that the positive answer will require some new geometric property imposed on $\partial M$, a sort of $\omega$ convexity.

It is natural to ask to what extend Calabi's characterization of intrinsically harmonic 1-forms $\omega$ and the results of this paper on the $\omega$-generated intrinsically near-minimal foliations extend to closed forms $\omega$ of any degree $k$. In particular, one might search for appropriate $k$-dimensional analogs of Calabi's positive loop condition, in other words, for a geometric characterization of the intrinsic harmonicity.

Recall that the rank of an exterior $k$-form $\omega: \Lambda^{k} V \rightarrow \mathbf{R}$ is defined to be the dimension of the space $\left.\{w\rfloor \omega, w \in \Lambda^{k-1} V\right\}$. Denote by $K_{\omega} \subset V$ the subspace defined by the equations $\{v\rfloor \omega=0\}_{v \in V}$ in $\Lambda^{k-1} V^{*}$. It is easy to verify that $\operatorname{dim} K_{\omega}+r k(\omega)=\operatorname{dim}(V)$. Also, if $r k(\omega)=k$, then $\omega$ is a product of 1-forms.

For a closed $k$-form $\omega$ of a constant rank, by a theorem of Cartan, the kernels $K_{\omega}$ form an integrable distribution. As before, the appropriate foliation is denoted by $\mathcal{F}_{\omega}$. If at some point the rank drops, the foliation $\mathcal{F}_{\omega}$ develops singularities.

Definition 4.4. We say that a closed $k$-form $\omega$ of a rank $\leq k$ on a compact oriented $n$-manifold $M$ has an $\omega$-positive cycle property, if for each leaf component $\mathcal{L}$ of the $(n-k)$-dimensional singular foliation $\mathcal{F}_{\omega}$, there exists a compact orientable $k$-submanifold $N$, such that: 
(1) $N$ intersects $\mathcal{L}$ transversally at a non-singular point of $\mathcal{F}_{\omega}$.

(2) $N$ is closed, or along its boundary $\partial N \subset \partial M, N$ is transversal to $\partial M$.

(3) The restriction of $\omega$ on $N$ is strictly positive (equivalently, $N$ is transversal to $\left.\mathcal{F}_{\omega}\right)$.

It can be shown that, if such a cycle $N$ can be conducted through one nonsingular point in $\mathcal{L}$, then there exists an $\omega$-positive cycle with properties (1)-(3) through any other non-singular point of $\mathcal{L}$.

Unlike the harmonic 1-forms which do satisfy the positive 1-cycle property, we do not expect any harmonic $k$-form of rank $k$ to satisfy the positive cycle property automatically. To establish such a property could be challenging. The first interesting case is provided by a harmonic 2 -form of rank $\leq 2$ (i.e. locally a product of two exact 1-forms) on a 4-manifold.

It is very likely that the methods of this paper can establish the validity of

Conjecture 4.5. Let a closed $k$-form $\omega$ satisfy the following list of properties:

(1) $\omega$ has rank $k$ on an open set $M_{\omega}^{\circ} \subset M$; the rank of $\omega$ drops on the singular set $S_{\omega}=M \backslash M_{\omega}^{\circ}$.

(2) $\omega$ is exact in a neighborhood of $S_{\omega}$.

(3) $\omega$ is intrinsically harmonic in a neighborhood of $S_{\omega}$.

(4) $\omega$ possess the $\omega$-positive cycle property.

Then $\omega$ is intrinsically harmonic everywhere and the foliation $\mathcal{F}_{\omega}$ is nearminimal away from $S_{\omega}$.

\section{References}

[Ca] E. Calabi, An Intrinsic Characterization of Harmonic 1-Forms, Global Analysis, Papers in Honor of K. Kodaira, D. C. Spencer and S. Iyanaga, Eds., 1969, 101-117.

[EL] J. Eells and L. Lemair, A Report on Harmonic Maps, Bull. London Math. Soc. 10 (1978), $1-68$.

[F] M. Farber, Exactness of the Novikov Inequalities, Functional Anal. and Appl. 19 (1985), $40-48$.

[FKL] M. Farber, G. Katz and J. Levine, Morse Theory of Harmonic Forms, Topology 37 (1998), 469-483.

[Fe] H. Federer, The Singular Sets of Area Minimizing Rectifiable Currents with Codimension One and of Area Minimizing Flat Chains Modulo Two with Arbitrary Codimension, Bull. Amer. Math. Soc. 76 (1970), 767-771.

[HS] R. Hardt and L. Simon, A Seminar on Geometric Measure Theory, 117, DMV Seminar 7, Birkhäuser-Verlag, 1986.

[HL] R. Harvey and H. B. Lawson, Calibrated Foliations (Foliations and Mass-minimizing Currents), American Jour. of Math. 104 (3) (1982), 607-633.

[Ho] K. Honda, A Note on Morse Theory of Harmonic 1-Forms, Topology 38 (1) (1999), 223233.

[HT] M. D. Hvidsten and P. Tondeur, A Characterization of Harmonic Foliations by Variation of the Metric, Proceedings of AMS 98 (2) (1986), 359-362. 
[KT] F. W. Kamber and P. Tondeur, Foliations and Metrics, Differential Geometry Proceedings, Special Year, Maryland, 1981-82, Birkhäuser, Boston-Basel-Stuttgart, 1983.

[N] S. P. Novikov, Multi-valued Functions and Functionals Analogue of Morse Theory, Soviet Math. Doklady 24 (1981), 222-226.

[N1] S. P. Novikov, Hamiltonian Formalism and Multi-valued Analogue of Morse Theory, Russian Math. Surveys 37 (5) (1982), 1-56.

[N2] S. P. Novikov, Topology of Foliations (in Russian), Trudy Mosk. Math. Obsh. 14 (1965), 248-277 (A.M.S. translation 1967)

[RS] D. Ruelle and D. Sullivan, Currents, Flows and Diffeomorphisms, Topology 14 (4) (1975), 319-327.

[Si] P. Simones, On a Class of Minimal Cones in $\mathbf{R}^{n}$, Bull. Amer. Math. Soc. 80 (1974), 488-489.

[S] D. Sullivan, Cycles for the Study of Foliated Manifolds and Complex Manifolds, Inventiones Math. 36 (1976), 225-255.

[S1] D. Sullivan, A Foliation of Geodesics is Characterized by having no "Tangent Homologies", Jour. of Pure and Applied Algebra 13 (1978), 101-104.

[S2] D. Sullivan, A Cohomological Characterization of Foliations Consisting of Minimal Surfaces, Comment. Math. Helvetici 54 (1979), 218-223.

[Ta] T. Tamura, Topology of Foliations: an Introduction, AMS Translations 97 (1992).

[Ti] D. Tishler, On Fibering Certain Foliated Manifolds over $S^{1}$, Topology 9 (1970), 153-154.

Gabriel Katz

5 Bridle Path Circle

Framingham, MA 01701

U.S.A.

e-mail: gabrielkatz@rcn.com

(Received: January 24, 2000)

\section{(D) To access this journal online: \\ (40) http://www.birkhauser.ch}

